\title{
Structural effects of soft nanoparticulate ligands on trace metal complexation thermodynamics
}

\author{
Elise Rotureau, ${ }^{1,2 *}$, Yves Waldvogel, ${ }^{1,2}$ José P. Pinheiro, ${ }^{1,2}$ José Paulo S. Farinha, ${ }^{3}$ Isabelle Bihannic, ${ }^{1,2}$ \\ Romain M. Présent, ${ }^{1,2}$ and Jérôme F. L. Duval ${ }^{1,2}$
}

1 CNRS, LIEC (Laboratoire interdisciplinaire des Environnements Continentaux), UMR 7360, Vandoeuvre-lès-Nancy F-54501, France

${ }^{2}$ Université de Lorraine, LIEC, UMR 7360, Vandoeuvre-lès-Nancy F-54501, France

${ }^{3}$ Centro de Quimica Fisica Molecular and IN-Institute of Nanoscience and Nanotechnology, Instituto Superior Tecnico, University of Lisbon, 1049-001 Lisboa, Portugal

* Corresponding author: elise.rotureau@univ-lorraine.fr

\begin{abstract}
Metal binding to natural soft colloids is difficult to address due to the inherent heterogeneity of their reactive polyelectrolytic volume and the modifications of their shell structure following changes in e.g. solution $\mathrm{pH}$, salinity or temperature. In this work, we investigate the impacts of temperature- and salinity-mediated modifications of the shell structure of polymeric ligand nanoparticles on the thermodynamics of divalent metal ions $\mathrm{Cd}(\mathrm{II})$-complexation. The adopted particles consist of a glassy core decorated by a fine-tunable poly(N-isopropylacrylamid) anionic corona. According to synthesis, the charges originating from the metal binding carboxylic moieties supported by the corona chains are located preferentially either in the vicinity of the core or at the outer shell periphery (p(MA-N) and $\mathrm{p}(\mathrm{N}-\mathrm{AA})$ particles, respectively). Stability constants $\left(K_{\mathrm{ML}}\right)$ of cadmium-nanoparticle complexes are measured under different temperature and salinity conditions using electroanalytical techniques. The obtained $K_{\mathrm{ML}}$ is clearly impacted by the location of the carboxylic functional groups within the shell as $\mathrm{p}(\mathrm{MA}-\mathrm{N})$ leads to stronger nanoparticulate $\mathrm{Cd}$ complexes than $\mathrm{p}(\mathrm{N}-\mathrm{AA})$. The dependence of $K_{\mathrm{ML}}$ on solution salinity for $\mathrm{p}(\mathrm{N}-\mathrm{AA})$ is shown to be consistent with a binding of $\mathrm{Cd}$ to peripheral carboxylic groups driven by coulombic interactions (Eigen-Fuoss mechanism for ions-pairing) or with particle electrostatic features operating at the edge of the shell Donnan volume. For p(MA-N) particulate ligands, a scenario where metal binding occurs within the intraparticulate Donnan phase correctly reproduces the experimental findings. Careful analysis of electroanalytical data further evidences that complexation of metal ions by core-shell particles significantly differ according to the location and distribution of the metal-binding sites throughout the reactive shell. This complexation heterogeneity is basically enhanced with increasing temperature i.e. upon significant increase of particle shell shrinking, which suggests that the contraction of the reactive phase volume of the particulate ligands promotes cooperative metal binding effects.
\end{abstract}




\section{Introduction}

Natural colloids have a large propensity to interact with metallic ions due to their inherently large specific surface area they therefore play a major role all along the biogeochemical cycle of trace metals in natural waters. Under environmental conditions, colloidal matter is characterized by a broad diversity of sizes, geometries, charges and chemical compositions thus offering a large spectrum of stability constants and association/dissociation rate constants of particulate metal complexes in natural aquatic systems. ${ }^{1-4}$ Over the last few years, significant efforts have been undertaken to elaborate a quantitative understanding of the chemodynamic features of (nano)particulate metal complexes with account of the physicochemical particle properties including their size or their functional groups density and distribution. ${ }^{5-7}$ As a prerequisite to such an investigation, an adequate physicochemical description of the reactive colloidal surface is obviously of paramount importance. A defining feature of most natural colloids (e.g. humic acids, ${ }^{8}$ microorganisms ${ }^{9-11}$ or polysaccharides ${ }^{12}$ ) is the diffuse nature of their soft interphase, i.e. the gradual distribution of their polymeric-like material from the inner part of the particle to the outer electrolyte solution. ${ }^{13}$ The softness degree of the particles basically defines the type of distributions of the charged functional groups, varying from a surface-like repartition in the limiting case of hard particles to a three dimensional distribution for soft porous particles. In this latter situation, the spatial confinement of functional groups acting as metal binding sites within the porous particle body has dramatic implications on the association/dissociation rates and stability of particle metal complexes. ${ }^{6}$ In particular, recent approaches revisited and extended the conceptual Eigen framework for metal complex formation with molecular ligands ${ }^{14}$ to the case of soft colloidal binding entities and proposed a scheme for metal complexation ${ }^{15-18}$ which comprises the following processes: (i) the diffusive/conductive transport of free hydrated metal ions $\left(\mathrm{M}^{\mathrm{z}+}\right)$ from bulk solution to particle/solution interface, (ii) the transport of $\mathrm{M}^{\mathrm{z+}}$ within the particle body towards the metal binding sites carried by the particle, (iii) followed by the formation of an outer-sphere reactant pair, and finally (iv) the release of water from the inner sphere of the metal ion to form a coordination bond with binding sites. This extended Eigen formalism allows the identification of the rate-limiting step (transport versus chemical kinetics) of the overall reactive pathway from free metal ions in bulk solution to complexed particulate metal form, according to various physico-chemical parameters such as particle size ${ }^{5-7,16,19,20}$ and softness, ${ }^{20-22}$ particle charge density, ${ }^{16,21}$ or medium ionic strength. ${ }^{16,21}$

Although such approaches have played a key role in our understanding of dynamic interactions between soft particles and metal ions, several questions regarding the impacts of soft particle structure heterogeneity on (thermo)dynamics of their metal complexation remain open. Such chemical and/or physical heterogeneity features will indeed give rise at the scale of a single particle to a distribution of stability constants of intraparticulate metal complexes or, equivalently, a binding site occupation that depends on the metal/complexing site ratio. ${ }^{23-25}$ Chemical heterogeneity originates from the polyfunctionality (e.g. various types of complexing sites) and polyelectrolytic features (e.g. presence of electric charges) of soft colloidal interfaces. ${ }^{25}$ Physical heterogeneity generally refers to the 
geometry of the particle and to the anisotropic arrangement of the material composing the particle throughout its volume. For the sake of illustration, fulvic acids exhibit a heterogeneous assemblage of aromatic and aliphatic components that directly impact the distribution of their functional sites and therewith their metal binding properties. ${ }^{26}$ Soft polymeric-like interphases are prone to structural heterogeneity also because of the generally distinct conformational properties of the chains located with the soft colloidal volume compared to those facing the external aqueous solution. ${ }^{12,27}$ In addition, structural modifications of soft particle interphase can occur following changes in the physicochemical medium conditions as a result of swelling/shrinking processes depending on $\mathrm{pH}$, ionic strength, temperature or solvent polarity. ${ }^{11,12}$ It is timely to analyse in some details the effects of structural organization of reactive soft colloidal interphases on their metal binding properties. This task is all the more urgent as the generic particle heterogeneity described above is seldom integrated within analysis of metal speciation in suspensions of soft particles. In view of these elements, the objective of this work is to measure and properly rationalize the relationship between structural organization of soft colloidal interphase and thermodynamics of its complexation of metal ions. To achieve this objective, the binding of metal ions by a well-characterized soft, charged nanoparticle is investigated here. The core-shell particles adopted here as surrogate for natural colloids consist of a glassy core of poly(methyl methacrylate) (PMMA) supporting a shell of polymer brushes made of poly(Nisopropylacrylamid) (PNIPAM) copolymerized with either poly(acrylic acid) or poly(methacrylic acid), hereafter referred to as $\mathrm{p}(\mathrm{N}-\mathrm{AA})$ and $\mathrm{p}(\mathrm{MA}-\mathrm{N})$ nanoparticles, respectively. As extensively detailed in our previous study, ${ }^{28}$ these two types of particles differ in terms of location of the carboxylic groups: $\mathrm{p}(\mathrm{N}-\mathrm{AA})$ carboxylic sites are distributed preferentially at the periphery of the particle whereas those for $\mathrm{p}(\mathrm{MA}-\mathrm{N})$ particle type are confined in the vicinity of the central particle core. Besides this feature, the PNIPAM-based shell of $\mathrm{p}(\mathrm{N}-\mathrm{AA})$ and $\mathrm{p}(\mathrm{MA}-\mathrm{N})$ nanoparticles is multiresponsive as its thickness depends on various stimuli, such as temperature, $\mathrm{pH}$ and solution ionic strength. ${ }^{28,29}$ The remarkable electrostatic and structural properties of $\mathrm{p}(\mathrm{N}-\mathrm{AA})$ and $\mathrm{p}(\mathrm{MA}-\mathrm{N})$ particles have been recently addressed in our previous work as a function of temperature, $\mathrm{pH}$ and ionic strength using potentiometric titration, electrokinetics, dynamic light scattering and small angle neutron scattering. ${ }^{28}$ This study evidenced the presence of a two-phase shell structure comprising a high density layer condensed on the core surface and a peripheral diluted shell layer. The heterogeneous shell structure revealed in this study together with the distinct locations of the functional carboxylic groups in the two chosen particles types constitute a promising basis for deciphering the role of particle structure on metal binding. Accordingly, the reactivity of $\mathrm{p}(\mathrm{N}-\mathrm{AA})$ and $\mathrm{p}(\mathrm{MA}-\mathrm{N})$ particles with respect to cadmium (II) ions, is examined here with use of two complementary stripping electroanalytical techniques that are suitable for the evaluation of the thermodynamic stability of complexes formed between metal ions and particulate ligands. AGNES (Absence of Gradients and Nernstian Equilibrium Stripping) is employed for the direct determination of the free metal concentration, which in fine provides an estimation of the stability constants of the complexed metal 
species in solution. ${ }^{30}$ Stripping chronopotentiometry at scanned deposition potential (SSCP) is further used to address the apparent degree of metal binding heterogeneity. ${ }^{31,32}$ The analysis is performed under different temperature and salinity conditions -both controlling the extent of shell swelling, which offers a way to quantify the effects of particle shell structure modification on thermodynamics of particulate metal complex formation.

\section{Theoretical section}

\subsection{Metal complexation analysis by electroanalytical techniques (SSCP and AGNES)}

Stripping electroanalytical techniques have been used in trace metal speciation studies due to their extremely low detection limit and their almost unique ability to provide dynamic information on metal complexation processes. Generally the consumption of free metal ions at an electrode surface generates a diffusion layer where equilibrium between free metal ions and metal complexes is affected. In the vicinity of the electrode surface, the complexation is generally schemed as follows:

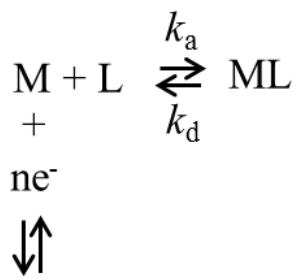

\section{$\mathrm{M}^{\circ}$}

, where ML stands for the metal complex formed with ligand $\mathrm{L}, n$ is the number of exchanged electrons between electrode and free metal ions $\mathrm{M}$, and $\mathrm{M}^{\circ}$ is the reduced form of $\mathrm{M}$. The constants $k_{\mathrm{a}, \mathrm{d}}$ correspond to the kinetic constants for ML formation and dissociation (subscripts a and $\mathrm{d}$, respectively). The stability constant of a given metal complex ML is defined by $K_{\mathrm{ML}}=k_{\mathrm{a}} / k_{\mathrm{d}}=$ $c_{\mathrm{ML}} /\left(c_{\mathrm{M}} c_{\mathrm{L}}\right)$ with $c_{\mathrm{M}}$ and $c_{\mathrm{ML}}$ the concentrations of free and complexed metal species in solution, respectively, and $c_{\mathrm{L}}$ is the free ligand concentration in solution. The association/dissociation rate constants $k_{\mathrm{a}}$ and $k_{\mathrm{d}}$ define the dynamic character of the complexation. Briefly, if the complexes are able to associate and dissociate while diffusing toward the electrode surface, they are deemed dynamic while if they are unable to do so they are called inert complexes. ${ }^{33}$ If a complex is dynamic, it may contribute to the amount of metal reduced at the electrode via its dissociation at the electrode surface. Dynamic complexes are labile if the association/dissociation kinetics are much faster than their diffusive transport, which in turn maintains an equilibrium between free and complexed metal concentrations at any time and position within the diffusion layer. In this case the totality of metal species in solution (free and complexed) are reduced. ${ }^{34}$

In the developments below, we briefly recall the basic theory used for the quantitative interpretation of SSCP data. The SSCP signal takes the form of a full voltammetric wave composed of the experimental set of $\left(\mathrm{E}_{\mathrm{d}}, \tau\right)$ couples with $\mathrm{E}_{\mathrm{d}}$ the applied deposition potential $(\mathrm{V})$ and $\tau$ the measured transition time 
(or electrolysis time in s). In situations where there is an excess of homogeneous ligands over metal ions, the stability constant $K_{\mathrm{ML}}$ can be retrieved from the shift $\Delta E_{1 / 2}$ between half-wave deposition potential measured in the presence and absence of ligands, irrespectively of the degree of lability of the formed metal complex, according to the DeFord-Hume equation: ${ }^{35}$

$\ln \left(1+K^{\prime}\right)=-(n F / R T) \Delta E_{1 / 2}-\ln \left(\tau_{\mathrm{M}+\mathrm{L}}^{*} / \tau_{\mathrm{M}}^{*}\right)$

, where $K^{\prime}=K_{\mathrm{ML}} c_{\mathrm{L}, \mathrm{T}}$. The electrolysis times $\tau_{\mathrm{M}}^{*}$ and $\tau_{\mathrm{M}+\mathrm{L}}^{*}$ in eqn (2) correspond to the limiting values of the wave heights obtained in the absence and presence of ligands, respectively, $R$ is the gas constant, $F$ the Faraday constant, and $T$ the temperature. In the presence of metal complexes with large ligands or loss of lability, the limiting transition time $\tau_{\mathrm{M}+\mathrm{L}}^{*}$ is lower than $\tau_{\mathrm{M}}^{*}$ since the supply of metal ions to the electrode surface decreases due to the formation of metal complexes in solution. For labile metal complexes, the overall metal flux at the electrode surface is fully controlled by the diffusion of free and complexed metal species from the solution, and the decrease in $\tau_{\mathrm{M}+\mathrm{L}}^{*}$ is then associated with the decrease in the mean diffusion coefficient of free and complexed metal species, recalling that for particulate ligands L we have $D_{\mathrm{ML}}<D_{\mathrm{M}}$ with $D_{\mathrm{ML}}$ and $D_{\mathrm{M}}$ the diffusion coefficients of the metal particulate complexes and of free metal ions, respectively. A metal complex stability constant $K^{\prime}$ function of the aforementioned decrease in the limiting transition time can be formulated using :

$$
\frac{\tau_{\mathrm{M}+\mathrm{L}}^{*}}{\tau_{\mathrm{M}}^{*}}=\left(\frac{\bar{D}}{D_{\mathrm{M}}}\right)^{p}=\left(\frac{1+\varepsilon K^{\prime}}{1+K^{\prime}}\right)^{p}
$$

, where $p$ is a parameter involving the contribution of hydrodynamics to metal mass transport from bulk solution to electrode surface, $\varepsilon$ is the diffusion coefficients ratio $D_{\mathrm{ML}} / D_{\mathrm{M}}$ and $\bar{D}$ the mean diffusion coefficient of M and ML species weighed by their respective concentrations in bulk solution. For a rotating disk electrode at the surface of which metal transfer is driven by coupled diffusion and convection, we have $p=2 / 3$, which is obtained from application of Levich's equation. ${ }^{36}$ For labile metal complexes, the stability constant $K^{\prime}$ from eqn (3) is equal to $K^{\prime}$ evaluated from eqn (2), while in case of non-labile systems, the flux is limited by the kinetics of complex dissociation at the electrode surface, and the application of eqn (3) then leads to an overestimation of the equilibrium complex stability constant. Consequently, a lability diagnosis can be elaborated from the comparison between the values of $K^{\prime}$ obtained from eqn (2) and (3). ${ }^{6}$ The reader is referred to references ${ }^{37-39}$ for further details on lability diagnostic.

As SSCP is a technique sensitive to the physicochemical dynamic conditions prevailing at the electrode surface, signal interpretation becomes necessarily more complicated for heterogeneous metal binding systems. It becomes then advantageous to collect information on metal complexes equilibrium properties in bulk solution, which can be done with use of AGNES technique. The latter is a stripping electrochemical technique that essentially performs potentiometric measurements in a mercury electrode by applying a deposition potential close to the standard reduction potential of the metal-Hg amalgam formation while waiting for a time delay (deposition time) sufficiently long to reach 
Nernstian equilibrium conditions. This strategy allows for a direct measurement of the free metal ion concentration in bulk solution exactly as an ion selective electrode (ISE), and for the same system as that used in dynamic SCP/SSCP experiments. ${ }^{30}$ From the measurement of $c_{\mathrm{M}}$ and the mass balance for the metal, one can then directly determine the stability constant $K_{\mathrm{ML}}$, irrespective of the lability degree or the heterogeneous features of the metal complexes. More details for the evaluation of the stability constants by AGNES technique are given in ESI.

\subsection{Metal binding heterogeneity analysis}

In cases where the solution contains metal complexes formed between metal ions and a single type of binding site, the slope $\left(\mathrm{d} \tau / \mathrm{d} E_{\mathrm{d}}\right)$ of the SSCP wave is defined by a Nernstian relationship. In contrast, for situations where the solution contains mixtures of metal complexes arising from heterogeneity of the metal binding particles, the description of the slope becomes more complicated. ${ }^{40}$ As each point of a given SSCP curve corresponds to an individual experiment performed at a given deposition potential, one probes metal complexes with distinct stability features all along the SSCP wave. In the foot of the wave, AGNES conditions are reached and one probes free metal ions only. Progressing toward more negative applied potentials, i.e. when approaching the top of the wave, more metal ions become reduced thus forcing the dissociation of stronger metal complexes which then contribute significantly to the measured electrochemical signal provided that they are dynamic. As a result, the SSCP wave obtained in the presence of metal complexes characterized by a distribution of stability constants, becomes spread out with respect to the potential axis as compared to that measured for the case of chemically homogeneous ligands. For this study, the magnitude of the heterogeneity degree in metal binding is evaluated from the deviation of the experimental SSCP wave against the homogeneous case, i.e. for one single type of ligand. This deviation is evaluated by comparing the full width at half maximum (FWHM) of the derivative plot $\left(\left(\mathrm{d} \tau / \mathrm{d} E_{\mathrm{d}}\right)\right.$ vs. $\left.E_{\mathrm{d}}\right)$ issued from the experimental curve with that corresponding to the homogeneous case (referring to the Nernstian law $\left.{ }^{37-39}\right)$, and given by $\Delta \alpha=\frac{(\mathrm{FWHM})^{\text {exp }}}{(\text { FWHM })^{\text {homogeneous }}}$. The corresponding ratio $\Delta \alpha$ provides an indicator of the heterogeneity extent of $\mathrm{Cd}$ binding with core-shell nanoparticles $(0<\Delta \alpha \leq 1$ with $\Delta \alpha=1$ pertaining to the homogeneous case). Prior to calculation of the derivative $\mathrm{d} \tau / \mathrm{d} E_{\mathrm{d}}$, the $\left(E_{\mathrm{d}}, \tau\right)$ plots were smoothed using curve fit function available in Kaleidagraph software.

\section{Materials and methods}

All solutions were prepared with ultrapure water (MilliQ, $18.2 \mathrm{M} \Omega \mathrm{cm}^{-1}$ ). Solutions of divalent cadmium $\mathrm{Cd}$ (II) were obtained from dilution of a $1000 \mathrm{ppm}$-certified standard solution (Fluka) of $\mathrm{Cd}\left(\mathrm{NO}_{3}\right)_{2}$. Solution ionic strength was adjusted with use of $1 \mathrm{M} \mathrm{NaNO}$ stock solution (Fluka, trace select 99.999\%), and diluted $\mathrm{HNO}_{3}$ or $\mathrm{NaOH}$ solutions (Merck suprapure, concentrations $0.1 \mathrm{M}$ or 
$0.01 \mathrm{M})$ were further used to adjust solution $\mathrm{pH}$. Stock solution of 3-(N-morpholino)propanesulfonic acid (MOPS, Sigma) was prepared from the corresponding solid powder and was used as pH buffer.

\subsection{Synthesis of multiresponsive core/shell particles}

The two core-shell particles adopted in this work were synthesized following the protocol detailed elsewhere. ${ }^{29,41}$ Briefly, the particles were prepared according to a two-stage shot emulsion copolymerization technique in ultrapure Milli-Q water. In the first step, the poly(methyl methacrylate) or PMMA core was prepared by batch emulsion polymerization, and in the second stage, a mixture of $N$-isopropylacrylamide (NIPAM) and methacrylic acid (MA) (for p(MA-N) particles) or acrylic acid (AA) (for $\mathrm{p}(\mathrm{N}-\mathrm{AA})$ particles) was added in three shots, $10 \mathrm{~min}$ apart, to the reactive medium at $70^{\circ} \mathrm{C}$, to form the shell around the PMMA core. Potassium persulfate (Aldrich, 99\% ACS reagent) was used for the initiator agent and sodium dodecyl sulfate (Sigma, 99\% GC grade) as the surfactant. The particles were cleaned by dialysis against Milli-Q water using a Spectra/Por membrane (MWCO of 6$8 \mathrm{kDa}$ ), renewing the water bath twice a day for two weeks. Due to the difference in reactivity ratios of the comonomers NIPAM/AA and NIPAM/MA, the comonomer distribution along the p(N-AA) chain differs compared with that of $\mathrm{p}(\mathrm{MA}-\mathrm{N})$ chain. For the $\mathrm{p}(\mathrm{N}-\mathrm{AA})$ the carboxylic groups are preferably located at the outer phase of the shell, whereas for $\mathrm{p}(\mathrm{MA}-\mathrm{N})$ the carboxylic groups are confined in the close vicinity of the particle core component. ${ }^{29}$

\subsection{Dynamic light scattering (DLS)}

Diffusion coefficient measurements of particles were measured with a Nano ZS Zetasizer from Malvern Instrument (He-Ne red laser source, $\lambda=633 \mathrm{~nm}$ ). The apparatus is equipped with a temperature-controlled quartz suprasil measurement cell, an automatic laser attenuator and an avalanche photodiode detector located at $173^{\circ}$ relative to the laser source, thus allowing backscattering detection. Experiments and data acquisition were driven by the Dispersion Technology Software from Malvern Instruments. Particles were dispersed at a final concentration of 0.005 vol. \% in $15 \mathrm{mM}$ and $100 \mathrm{mM} \mathrm{NaNO} 3$ electrolyte solutions at $\mathrm{pH}$ 7.5. Solution $\mathrm{pH}$ and salinity were fixed after addition of appropriate aliquots of $0.1 \mathrm{M} \mathrm{NaOH}, 0.1 \mathrm{M} \mathrm{HNO}_{3}$ and $1 \mathrm{M} \mathrm{NaNO}_{3}$ solutions. Prior to measurements at a given $\mathrm{pH}$ and salt concentration, particle dispersions were systematically pre-equilibrated at the desired temperature $\left(15,25\right.$ and $\left.35^{\circ} \mathrm{C}\right)$ for a period of at least $40 \mathrm{~min}$. Particle diffusion coefficients were derived from analysis of the measured intensity autocorrelation functions. As a first approximation, the corresponding monomodal (intensity-based) distributions in (hydrodynamic) size of equivalent hard spheres were evaluated on the basis of the Stokes-Einstein equation. These measurements complete those carried out at lower $\mathrm{pH}$ values reported in our previous study. ${ }^{28} \mathrm{We}$ systematically verified that measured particle size was not affected by the presence of metal ions 
present at the trace level under the conditions of interest in this work, in line with conclusions by Sagou et al. ${ }^{42}$

\subsection{Electrochemical analysis of metal complexation}

An Ecochemie Autolab type III potentiostat controlled by GPES 4.9 software (Ecochemie, The Netherlands) was used in conjunction with a Metrohm 663VA stand. The auxiliary electrode was a glassy carbon rod and the reference electrode was an $\mathrm{Ag} / \mathrm{AgCl}$ electrode. The working electrode was a thin mercury film electrode (TMFE) plated onto a rotating glassy carbon (GC) disk of $2 \mathrm{~mm}$ in diameter (Metrohm). The preparation of TMFE was repeated daily for each set of experiments. GC electrode was conditioned with a polishing and electrochemical pre-treatment according to the procedures reported elsewhere. ${ }^{43}$ The thin mercury film was plated ex-situ on the GC electrode with use of a $0.48 \mathrm{mM}$ mercury (II) nitrate solution under acidic conditions $\left(0.75 \mathrm{mM} \mathrm{HNO}_{3}, \mathrm{pH} 1.9\right)$ via electrodeposition at $-1.3 \mathrm{~V}, 1000 \mathrm{rpm}$ for 240s. Once the experiments were completed, the TMFE was cleaned using successive mercury reoxidations in $80 \mathrm{mM}$ ammonium thiocyanate solution buffered with ammonium acetate ( $\mathrm{pH} 3.4)$.

\section{Experimental protocol for electrochemical measurements.}

For each condition explored in this study, namely the nature of the particle, temperature and ionic strength, the experiments were conducted twice. A disposable polystyrene cell was placed in a doublewalled container connected to a refrigerating-heating water circulator in order to set temperature of particles suspensions at 15,25 or $35^{\circ} \mathrm{C}$ with an accuracy of $\pm 0.1^{\circ} \mathrm{C}$ during electrochemical measurements. Batch suspension of $0.1 \%$ (w/w) PNIPAM particles stabilized in $15 \mathrm{mM}$ or $100 \mathrm{mM}$ $\mathrm{NaNO}_{3}$ electrolyte, $5 \times 10^{-7} \mathrm{M} \mathrm{Cd}\left(\mathrm{NO}_{3}\right)_{2}$ and $1 \mathrm{mM}$ MOPS buffer ( $\mathrm{pH} 7.5$ ), was equilibrated at room temperature at least $24 \mathrm{~h}$ prior to measurements. The corresponding metal-to-ligand ratios are equal to 0.0016 for $\mathrm{p}(\mathrm{N}-\mathrm{AA})$ suspensions $\left(c_{\mathrm{L}, \mathrm{T}}=3 \times 10^{-4} \mathrm{M}\right)$ and 0.0018 for $\mathrm{p}(\mathrm{MA}-\mathrm{N})$ suspensions (with $c_{\mathrm{L}, \mathrm{T}}=2.7 \times 10^{-4} \mathrm{M}$ ). Calibration of the SSCP measurements $\left(E_{\mathrm{d}}\right.$ in the range -0.78 to $-0.65 \mathrm{~V} v s$. $\mathrm{Ag} / \mathrm{AgCl}$ electrode) was performed beforehand in a $5 \times 10^{-7} \mathrm{M} \mathrm{Cd}(\mathrm{II})$ solution acidified to $\mathrm{pH} \mathrm{3}$, in the absence of particles under ionic strength conditions similar to those pertaining to experiments conducted in the presence of nanoparticles. The deposition step was carried out at a specified deposition potential $E_{\mathrm{d}}$ for a time delay $t_{\mathrm{d}}$ of $45 \mathrm{~s}$ and an electrode rotation speed of 1500 $\mathrm{rpm}$. Then, a $3 \mu \mathrm{A}$ stripping current $I_{\mathrm{s}}$ was applied until the potential reached a value well beyond the reoxidation transition plateau ( $-0.4 \mathrm{~V}$ for $\mathrm{Cd})$.

AGNES measurements were performed following the hereafter detailed protocol. The metal deposition step at the $\mathrm{Hg}$ electrode was divided into two sub-stages: (i) first, a potential $E_{1, \mathrm{a}}$ corresponding to conditions where deposition process is diffusion-limited, was applied for a time $t_{1, \mathrm{a}}$ while stirring at a rotation speed of $1500 \mathrm{rpm}$, and (ii) a potential $E_{1, \mathrm{~b}}$ was then fixed for a delay $t_{1, \mathrm{~b}}$ set equal to $3 t_{1, \mathrm{a}}$ (with stirring) in order to establish equilibrium between metal concentrations at the 
electrode surface and in bulk solution. The magnitude of the potential $E_{1, \mathrm{a}}$ was chosen to coincide with the deposition potential at the limiting SSCP wave height (-0.75V vs $\mathrm{Ag} / \mathrm{AgCl}$ electrode for $\mathrm{Cd})$, and $E_{1, \mathrm{~b}}$ at the half wave potential of the SSCP curve. The characteristic times $t_{1, \mathrm{a}}$ and $t_{1, \mathrm{~b}}$ were fixed at $40 \mathrm{~s}$ and 120 s, respectively.

\section{Results and discussion}

\subsection{Salinity- and temperature-dependent size and structure of core-shell PMIPAM-based nanoparticles}

The charged PNIPAM copolymer brushes confer to the shell an outstanding thermo-, $\mathrm{pH}-$ and ionoresponsiveness. From our previous work, ${ }^{28}$ the overall particle radius (including both particle core and shell compartment) can increase from $50 \pm 5 \mathrm{~nm}$ in the collapsed shell state, at temperature above $35^{\circ} \mathrm{C}$ at $\mathrm{pH} 4$ and $15 \mathrm{mM}$ solution ionic strength, to $110 \pm 5 \mathrm{~nm}$ in the fully expanded (swollen) shell state at temperature below $25^{\circ} \mathrm{C}$ at $\mathrm{pH}$ 7.5. Small angle neutron scattering (SANS) and DLS data analysis evidenced the presence of two polymeric phases in the shell, namely a high-density layer condensed on the core surface (detected by SANS) and a low-density polymer brush expanded towards the bulk solution (detected by DLS) at sufficiently low temperatures, which comforts theoretical modeling highlighting a non-ideal brush behaviour for these particulate core-shell systems ${ }^{41}$ SANS study further revealed that the thickness of the condensed shell phase increases from 5 to $10 \mathrm{~nm}$ with changing temperature from 15 to $45^{\circ} \mathrm{C}$. This size variation stems from the collapse of the peripheral diluted shell layer upon increasing temperature. In addition, particle electrokinetic and titration studies support the preferential location of the carboxylic groups at the vicinity of the core and at the periphery of the shell for the $\mathrm{p}(\mathrm{MA}-\mathrm{N})$ and $\mathrm{p}(\mathrm{N}-\mathrm{AA})$ particle types, respectively. For $\mathrm{p}(\mathrm{MA}-\mathrm{N})$ particles, charges at the vicinity of the core are likely exposed to hydrophobic patches and accordingly, p(MA-N) nanoparticle exhibits delayed dissociation of the carboxyl groups with $\mathrm{pH}$ as compared to that of $\mathrm{p}(\mathrm{N}-\mathrm{AA})$ nanoparticles. ${ }^{28}$ 


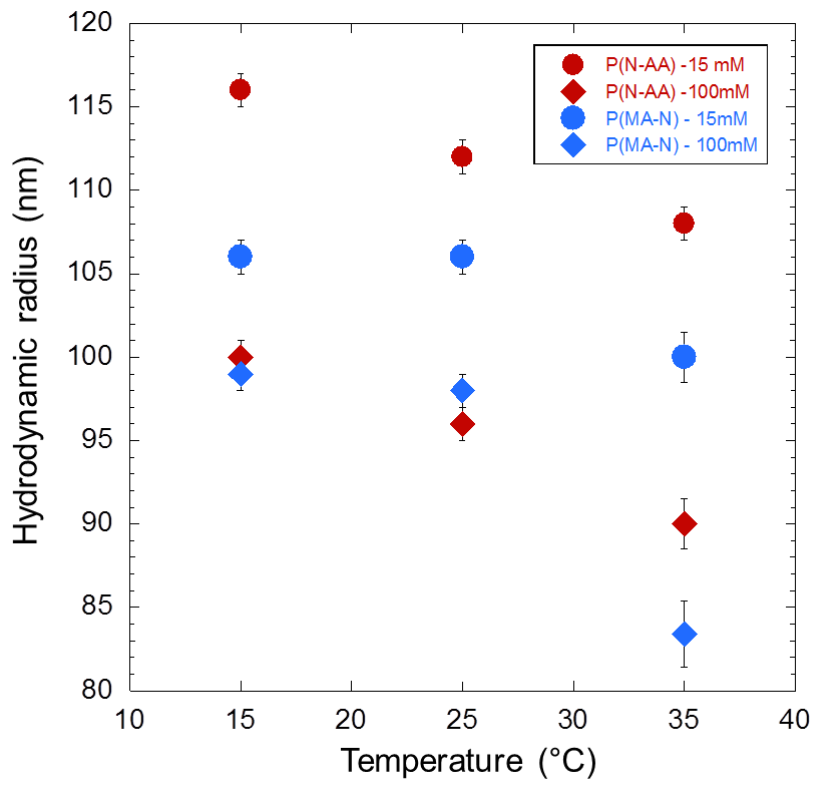

Figure 1: Hydrodynamic radius $R_{\mathrm{H}}$ of $\mathrm{p}(\mathrm{MA}-\mathrm{N})$ and $\mathrm{p}(\mathrm{N}-\mathrm{AA})$ particles computed on the basis of StokesEinstein equation using particle diffusion coefficients obtained by dynamic light scattering (DLS) as a function of temperature, at $\mathrm{pH}$ 7.5, $15 \mathrm{mM}$ and $100 \mathrm{mM}$ electrolyte concentrations (indicated).

The current section focuses on the swelling properties of the multi-responsive particles at $\mathrm{pH} 7.5$, which corresponds to the $\mathrm{pH}$ condition of the electroanalytical metal-binding experiments detailed later. Figure 1 displays the temperature dependence of the hydrodynamic radius $R_{\mathrm{H}}$ of $\mathrm{p}(\mathrm{MA}-\mathrm{N})$ and $\mathrm{p}(\mathrm{N}-\mathrm{AA})$ particles as determined by DLS in $15 \mathrm{mM}$ and $100 \mathrm{mM} \mathrm{NaNO}_{3}$ solution at $\mathrm{pH}$ 7.5. For both particles examined, $R_{\mathrm{H}}$ slightly decreases with increasing temperature from $15^{\circ} \mathrm{C}$ to $35^{\circ} \mathrm{C}$. This particle shell shrinking, well-documented for thermoresponsive colloidal systems, is less significant than that measured at lower $\mathrm{pH}$ values where magnitude of repulsive interactions between intraparticulate ionogenic charges is lower. ${ }^{28}$ At $\mathrm{pH} 7.5$, previously published titration data ${ }^{28}$ evidenced that the carboxylic groups located in the shell of $\mathrm{p}(\mathrm{N}-\mathrm{AA})$ particles are fully deprotonated while their dissociation in $\mathrm{p}(\mathrm{MA}-\mathrm{N})$ particles shell is not complete. For the latter particle type, $83 \%$ and $90 \%$ of the total amount of carboxylic groups are deprotonated in $15 \mathrm{mM}$ and $100 \mathrm{mM} \mathrm{NaNO} 3(\mathrm{pH} 7.5$ ), respectively. Due to the presence of repulsive electrostatic interactions originating from the charges carried by the particle core supported-polymer brushes, the lower critical solution temperature (LCST) is shifted to larger temperature values compared to that typically measured for uncharged PNIPAM polymer, generally between $32-37^{\circ} \mathrm{C}$. In the temperature range of interest in this work $\left(15^{\circ} \mathrm{C}-35^{\circ} \mathrm{C}\right)$, the polymer chains within the dilute shell phase are significantly expanded, and the particle size is much larger than that found at lower $\mathrm{pH}$ and temperature well above the LCST where shell collapse is achieved $\left(R_{\mathrm{H}}=50 \pm 5 \mathrm{~nm}^{28}\right)$.

At fixed temperature, the shell tends to shrink upon increasing solution salinity due to classical screening effects. ${ }^{28}$ At $15 \mathrm{mM}$, we note that $\mathrm{p}(\mathrm{N}-\mathrm{AA})$ particles radius is $c a .10 \mathrm{~nm}$ larger than that of $\mathrm{p}(\mathrm{MA}-\mathrm{N})$ particles. This reflects the differentiated location of carboxylic moieties in the shell of these 
two particles types. For $\mathrm{p}(\mathrm{N}-\mathrm{AA})$, according to the synthesis procedure charges are preferably positioned at the periphery of the shell so that under conditions where repulsive interactions take place, polymer brushes can freely expand towards bulk solution. This contrasts with $\mathrm{p}(\mathrm{MA}-\mathrm{N})$ for which the intra-shell repulsive forces are operational mainly in the direct vicinity of the particle core where charges are predominantly distributed. ${ }^{28,29}$ At $100 \mathrm{mM}$ ionic strength, particle charge screening is quite effective for both particles, which leads to dampening of the difference in sizes related to the charge distribution in the particle shell. It is emphasized that at $35^{\circ} \mathrm{C}$ and $100 \mathrm{mM} \mathrm{NaNO}$, p(MA-N) particles exhibit a stronger collapse than $\mathrm{p}(\mathrm{N}-\mathrm{AA})$, which suggests more significant intermolecular hydrophobic interactions for the former particle type under such conditions.

\subsection{Thermodynamic stability of soft nanoparticulate metal complexes}

Metal complexation studies were performed at pH 7.5 in $15 \mathrm{mM}$ and $100 \mathrm{mM}$ salt concentration at $15^{\circ} \mathrm{C}, 25^{\circ} \mathrm{C}$ and $35^{\circ} \mathrm{C}$. For each condition examined, we performed AGNES measurements and evaluated free metal concentration in solution and therewith the nanoparticulate metal complex stability constant. The apparent $K_{\mathrm{ML}}$ values are defined in terms of the average concentrations of the free and complexed species in the dispersion, including the electrostatic contributions to metal binding by nanoparticulate ligands. The so-obtained $K_{\mathrm{ML}}$ constants are collected in Table 1.

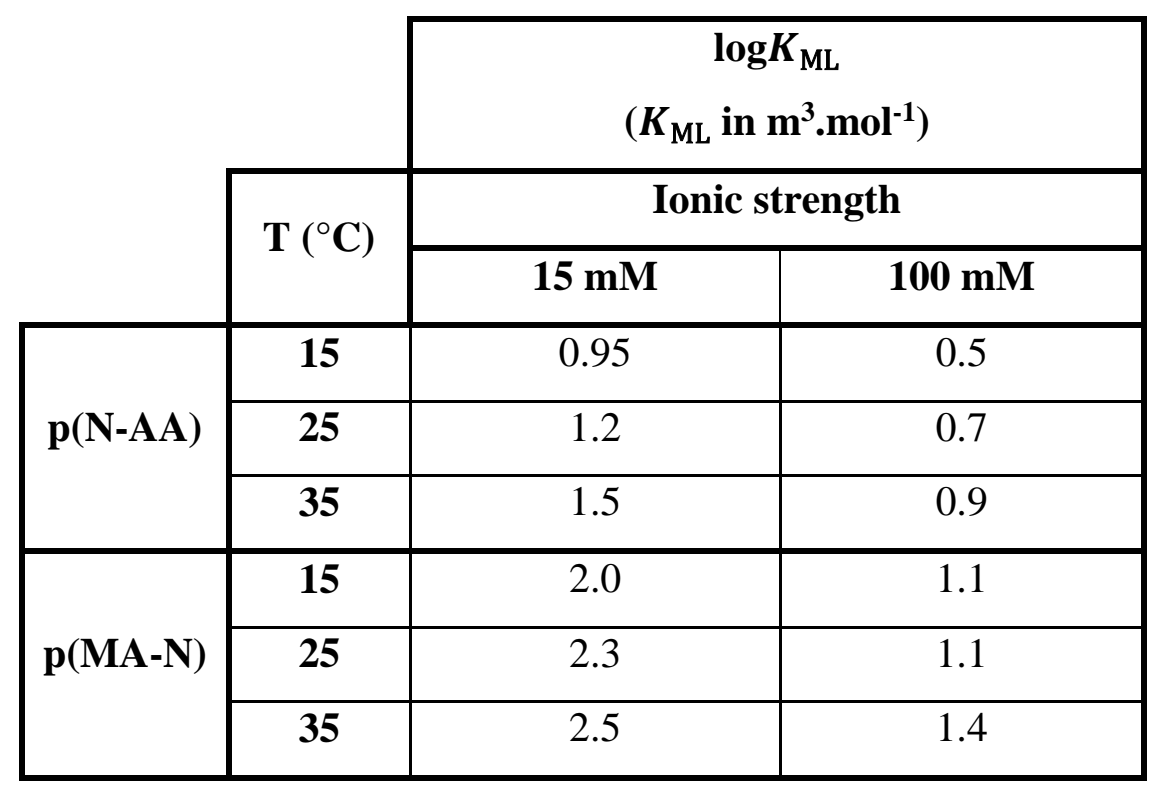

Table 1: Stability constants $K_{\mathrm{ML}}$ for soft nanoparticulate Cd(II)-complexes evaluated from AGNES analysis at $\mathrm{pH}$ 7.5. The values of $K_{\mathrm{ML}}$ correspond to the means of three measurements.

The results evidence that the stability of Cd-p(MA-N) particle complexes is systematically larger than that obtained with $\mathrm{p}(\mathrm{N}-\mathrm{AA})$ particles, irrespective of the solution ionic strength and temperature conditions tested. At $15 \mathrm{mM}$ ionic strength, one order of magnitude differentiates the log values of 
$K_{\mathrm{ML}}$ obtained for the two particle types, while at $100 \mathrm{mM}$ solution ionic strength, a 0.4-0.6 difference is obtained depending on temperature. The stability constants calculated at $100 \mathrm{mM} \mathrm{NaNO}$ concentration are of specific interest since the $\mathrm{p}(\mathrm{MA}-\mathrm{N})$ and $\mathrm{p}(\mathrm{N}-\mathrm{AA})$ particles radius and carboxylic dissociation features are comparable at such high salinity level (Figure 1) The difference observed between stability constants for these two particles under such salt concentration conditions reflects a significant role of the differentiated spatial distribution of the $-\mathrm{COOH}$ sites within the shell on their reactivity toward metal ions. In particular, the stronger stability values $K_{\mathrm{ML}}$ evaluated with $\mathrm{p}(\mathrm{MA}-\mathrm{N})$ as particulate ligands may evidence a cooperative effect of carboxylate moieties in metal binding, recalling that the charges significantly reside in the proximity of $\mathrm{p}(\mathrm{MA}-\mathrm{N})$ particle core and are therefore likely more condensed than $\mathrm{p}(\mathrm{N}-\mathrm{AA})$ charges, the latter being significantly pushed according to synthesis- to the shell periphery. ${ }^{29}$ A quantitative analysis of this issue will be detailed in the discussion section from proper examination of the electrostatic contribution to $K_{\mathrm{ML}}$. On a qualitative level, increasing the solution ionic strength impacts metal binding via screening of the (negative) particle charges, which results in a reduction of the stability constant of the formed metal complexes. Our results are clearly consistent with this anticipation.
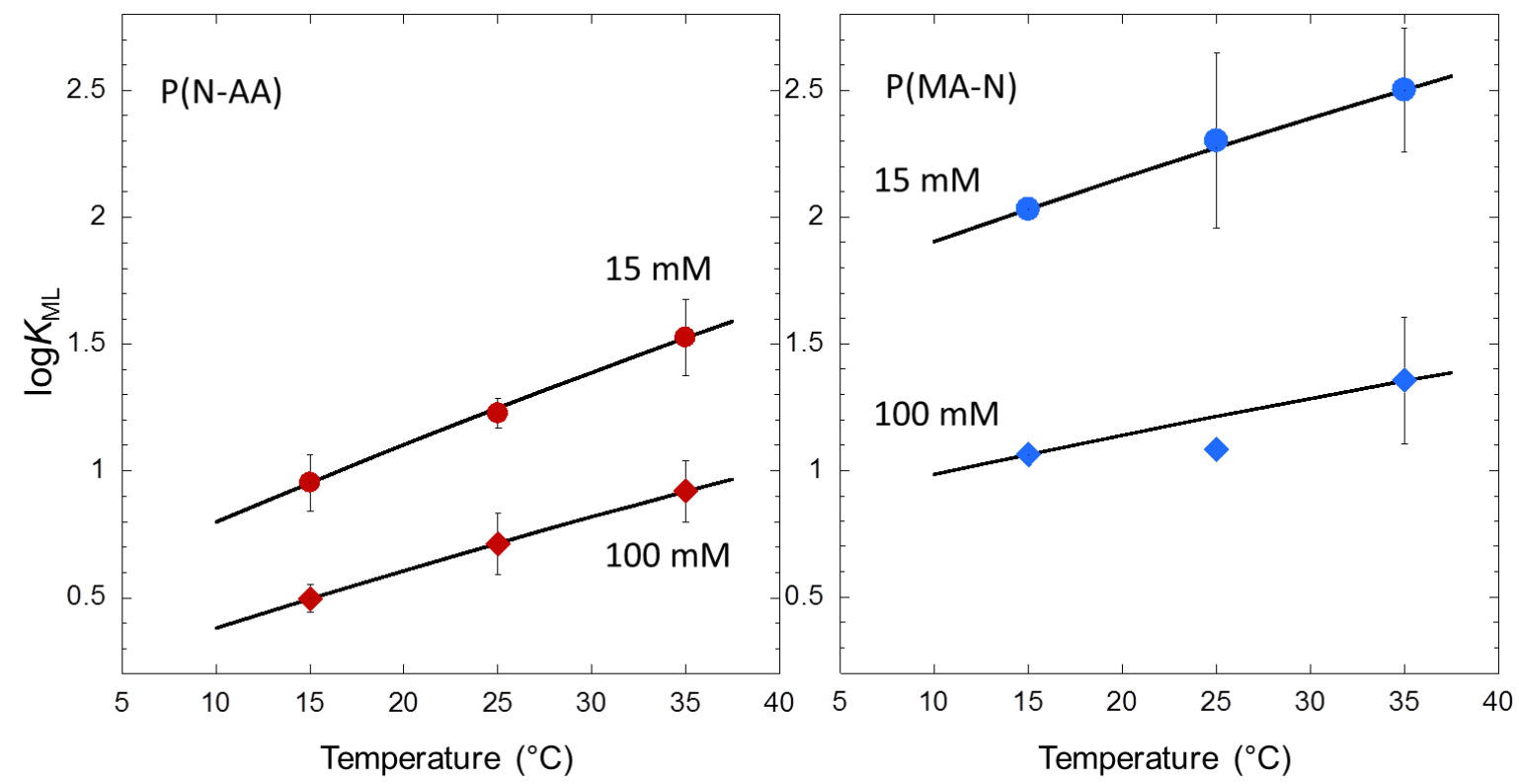

Figure 2: Temperature dependence of the stability constants $K_{\mathrm{ML}}$ for the two core-shell particles (indicated) determined by AGNES technique. The solid line represents the Gibbs-Helmholtz relationship using the value of the standard enthalpy of reaction $\Delta H^{0}$ and the entropy change $\Delta S^{0}$ given in Table 2. The values of $K_{\mathrm{ML}}$ correspond to the means of three measurements with the uncertainties calculated from the standard deviation.

Figure 2 reports the dependence of the ML stability constants on temperature at fixed salt concentration for both particle types considered in this work. The standard enthalpy of reaction $\Delta H^{0}$ is determined from the van't Hoff equation given by

$\frac{\mathrm{d} \ln K_{\mathrm{ML}}}{\mathrm{d} T}=\frac{\Delta H^{0}}{R T^{2}}$ 
, and the entropy change $\Delta S^{0}$ is evaluated from the basic Gibbs - Helmholtz equation that connects $K_{\mathrm{ML}}$ to temperature $T$ according to:

$\Delta G^{0}=\Delta H^{0}-T \Delta S^{0}=-R T \ln K_{\mathrm{ML}}$.

The obtained values of $\Delta H^{0}$ and $\Delta S^{0}$ are listed in Table 2 and the ensuing Gibbs-Helmholtz relationships are displayed in Figure 2 for the sake of comparison with experiments.

\begin{tabular}{|c|c|c|c|c|}
\hline Ionic strength & \multicolumn{2}{|c|}{$15 \mathrm{mM}$} & \multicolumn{2}{|c|}{$100 \mathrm{mM}$} \\
\hline & $\Delta H^{0}(\mathrm{~kJ} / \mathrm{mol})$ & $\Delta S^{0}\left(\mathrm{~J}^{\prime} \mathrm{mol}^{-1} \cdot \mathrm{K}^{-1}\right)$ & $\Delta H^{0}(\mathrm{~kJ} / \mathrm{mol})$ & $\Delta S^{0}\left(\mathrm{~J}^{\prime} \mathrm{mol}^{-1} \cdot \mathrm{K}^{-1}\right)$ \\
\hline $\mathbf{p}(\mathbf{N}-\mathbf{A A})$ & 48.5 & 186.5 & 36.0 & 134.5 \\
\hline $\mathbf{p}(\mathbf{M A}-\mathbf{N})$ & 40.0 & 177.8 & 24.7 & 105.1 \\
\hline
\end{tabular}

Table 2: Standard enthalpy $\Delta H^{0}$ and entropy change $\Delta S^{0}$ associated with the complex formation reaction between cadmium ions and $\mathrm{p}(\mathrm{N}-\mathrm{AA})$ or $\mathrm{p}(\mathrm{MA}-\mathrm{N})$ ligand particles in $15 \mathrm{mM}$ and $100 \mathrm{mM}$ salt concentrations.

The sign of $\Delta H^{0}$ values evidences an endothermic metal-nanoparticle complex formation reaction that is promoted with increasing temperature. This contrasts with the exothermic formation of metal complexes with small or molecular ligands like nitrilotriacetic acid. ${ }^{44}$ Both $\Delta H^{0}$ and $\Delta S^{0}$ are positive, which means that metal complex formation is enthalpically unfavourable and is driven by an increase in entropy. The gain in entropy may result from a favourable change in the hydration states of metallic ions upon binding due to the release of water from their hydration sphere and to the replacement of two monovalent ions by one divalent ion in the polymeric shell phase, as described in ${ }^{45}$ and ${ }^{46}$. The disparities between entropy and energy values obtained for the two ionic strengths or two particle types tested are not easy to interpret as several processes likely take place when changing salt concentration and/or particle structure, e.g. modification in intraparticulate solvent structure and underlying effects on (i) complex formation, (ii) polymer chains configuration and (iii) metal coordination number.

\subsection{Temperature-dependent heterogeneity in metal binding by core-shell PNIPAM-based particles}

To go further in the evaluation of the metal binding properties of $\mathrm{p}(\mathrm{N}-\mathrm{AA})$ and $\mathrm{p}(\mathrm{MA}-\mathrm{N})$ soft particles, SSCP analysis was carried out under $\mathrm{pH}$ and temperature conditions similar to those mentioned in Table 1. Illustrative SSCP measurements are given in Figure 3 for metal binding by $\mathrm{p}(\mathrm{N}-$ AA) particles in $15 \mathrm{mM}$ salt concentration solution at $15^{\circ} \mathrm{C}, 25^{\circ} \mathrm{C}$ and $35^{\circ} \mathrm{C}$. The qualitatively similar SSCP results for binding of $\mathrm{Cd}(\mathrm{II})$ by $\mathrm{p}(\mathrm{N}-\mathrm{AA})$ at $100 \mathrm{mM}$ ionic strength and $\mathrm{p}(\mathrm{MA}-\mathrm{N})$ particles at 15 and $100 \mathrm{mM}$ ionic strengths are given in ESI. 

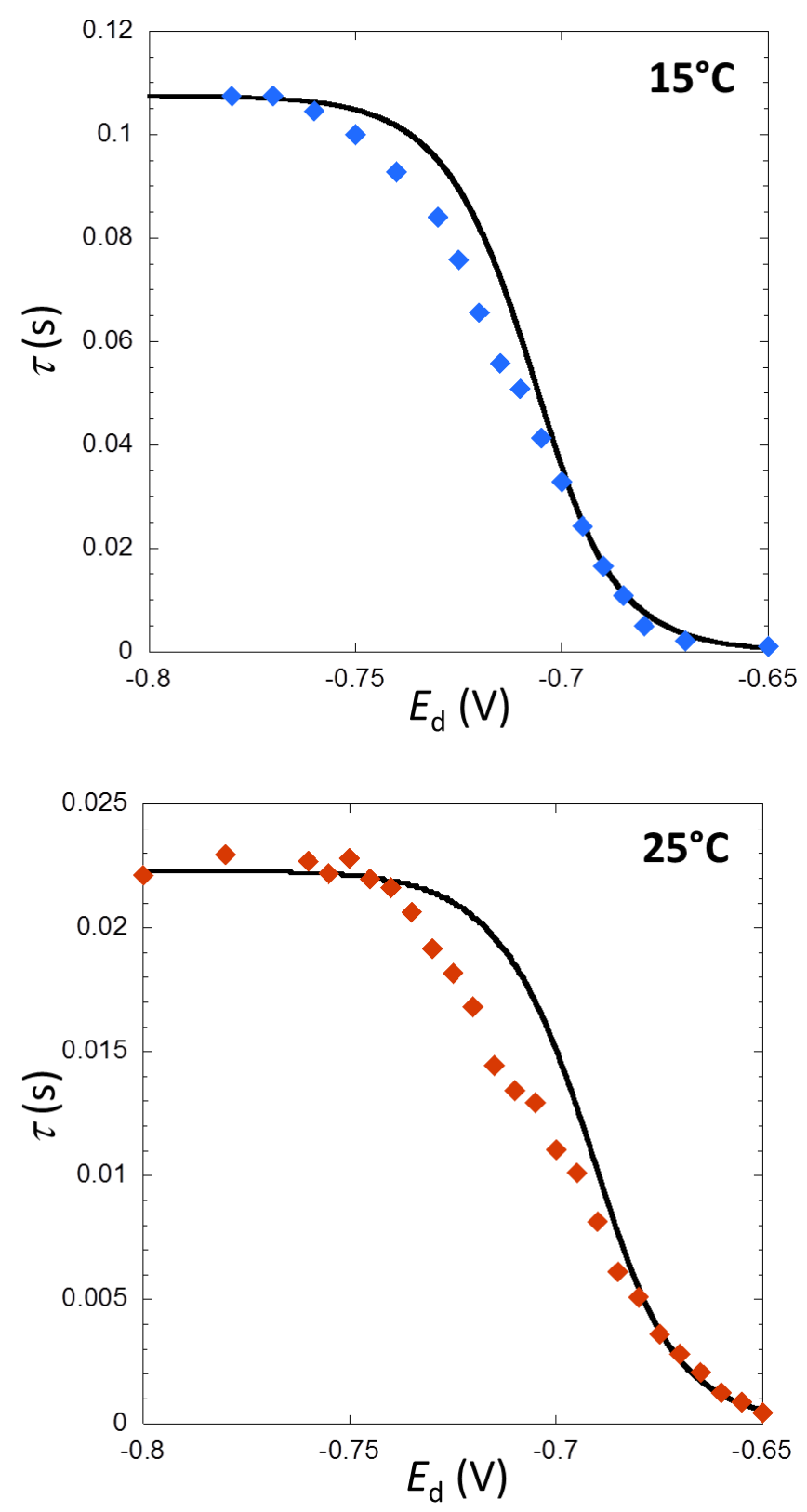


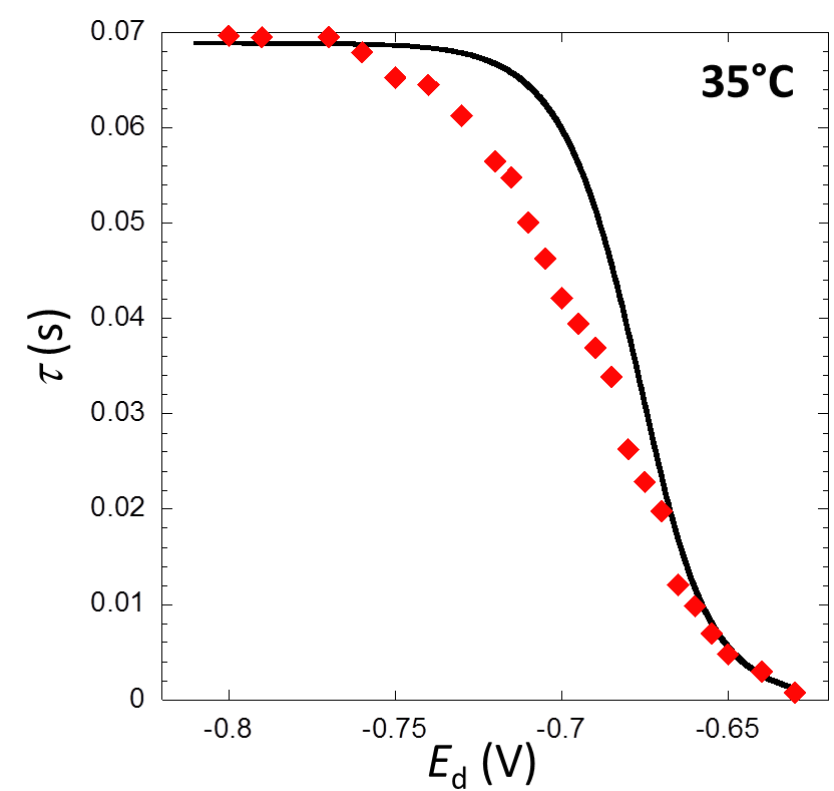

Figure 3: SSCP experimental points and simulated curves in the presence of $\mathrm{Cd}$ (total concentration of $5 \times 10^{-7}$ $\mathrm{M})$ and $\mathrm{p}(\mathrm{N}-\mathrm{AA})$ particles at $0.1 \%(\mathrm{w} / \mathrm{w})$. Experiments were conducted at $15 \mathrm{mM}$ ionic strength and $\mathrm{pH} 7.5$. Solid lines represent the simulated curves for homogeneous metal complexation (see text for details).

\section{Full-wave SSCP analysis: heterogeneity evidences and lability diagnosis}

Experimental SSCP data were analysed according to the theory detailed in ${ }^{34}$ that is valid for a single type of ligand. In details, we attempted to fit the SSCP curves by adjusting the potential shift $\Delta E$ in order to minimize the difference between the thermodynamic constant $K^{\prime}$ as derived from eqn (2) and that obtained from AGNES analysis. The resulting computed SSCP waves are reported in Figure 3 and in Figure S1 in ESI. As shown in Figure 3, the theory successfully reproduces the lower part of the experimental SSCP waves corresponding to the contribution of free metal ions, but it fails to recover the rising portion of the wave. As detailed in $\$ 2.2$, the discrepancy between the rising portion of the experimental SSCP wave and that evaluated for the homogeneous binding situation evidences heterogeneous metal complexation between metal ions and particles where functional groups with various reactivities/affinities towards the metal ions are involved. Accordingly, the next section is devoted to an in-depth comparison of the heterogeneity features of Cd complexation by the hereadopted core-shell nanoparticles as a function of temperature and medium salt content.

For all SSCP results obtained in this work as a function of the type of particulate ligand, the solution temperature and the electrolyte concentration, fitting of experimental data at the foot of the SSCP wave was systematically achieved with equal values of $K^{\prime}$ derived from eqns (2) and (3). This evidences the labile nature of the probed metal complexes. Of course, in case of heterogeneous complexation scenario, a decrease in the complex lability upon approaching the top of the wave i.e. with applying more negative potentials, can not be ruled out. However, having in mind that the estimation of $K^{\prime}$ from eqn (3) involves the SSCP plateau value that originates from contributions of all 
metal species probed by the electrode, it is legitimate to argue that our lability diagnosis pertaining to complexes probed at the foot of the wave applies all along the SSCP curve..

\section{Metal binding heterogeneity analysis}

This section is devoted to a quantitative analysis of SSCP data in order to determine the extent of heterogeneity in particle metal binding sites as a function of the type of particulate ligands, temperature and electrolyte concentration. The obtained heterogeneity parameters $\Delta \alpha$ defined in $\S 2.2$. are collected in Table 3.

\begin{tabular}{|c|c|c|c|}
\cline { 2 - 4 } \multicolumn{1}{c|}{} & \multicolumn{2}{c|}{$\Delta \alpha$} \\
\cline { 2 - 4 } \multicolumn{1}{c|}{} & $\mathbf{T}\left({ }^{\circ} \mathbf{C}\right)$ & $\mathbf{1 5} \mathbf{~ m M}$ & $\mathbf{1 0 0} \mathbf{~ I M}$ \\
\cline { 2 - 4 } & $\mathbf{1 5}$ & $0.66 \pm 0.2$ & $0.80 \pm 0.2$ \\
\cline { 2 - 4 } $\mathbf{4}$ p(N-AA) & $\mathbf{2 5}$ & $0.57 \pm 0.2$ & $0.79 \pm 0.2$ \\
\cline { 2 - 4 } & $\mathbf{3 5}$ & $0.45 \pm 0.2$ & $0.70 \pm 0.2$ \\
\hline \multirow{3}{*}{$\mathbf{p}(\mathbf{M A}-\mathbf{N})$} & $\mathbf{1 5}$ & $0.60 \pm 0.3$ & $0.79 \pm 0.2$ \\
\cline { 2 - 4 } & $\mathbf{2 5}$ & $0.56 \pm 0.2$ & $0.70 \pm 0.3$ \\
\cline { 2 - 4 } & $\mathbf{3 5}$ & $0.38 \pm 0.4$ & $0.50 \pm 0.4$ \\
\hline
\end{tabular}

Table 3: Heterogeneity parameter $(\Delta \alpha)$ derived from the ratio between the full width at half maximum of the experimental $\left(\mathrm{d} \tau / \mathrm{d} E_{\mathrm{d}}\right)$ plot and that corresponding to the homogeneous complexation situation.

The nature of the heterogeneity in metal binding is not straightforward to identify as it may originate from different (and intertwined) processes, e.g. the possible formation of complexes with different stoichiometries involving carboxylic groups and/or the lone pair electrons of nitrogen atom, and the distinct chemical environments of the metal binding sites depending on their position across the shell. This latter option likely applies for the core-shell systems of interest, recalling that according to their position and distribution along the chains, carboxylate moieties are more or less exposed to hydrophobic patches: groups protruding towards the outer shell are more hydrated than those close to the core in the condensed polymeric phase. Overall, and in line with this argument, $\mathrm{Cd}$ binding by $\mathrm{p}(\mathrm{MA}-\mathrm{N})$ particles displays a more pronounced heterogeneity than that by $\mathrm{p}(\mathrm{N}-\mathrm{AA})$. At this stage, it is worth mentioning that there is an inherent interplay between complex stability strength and metal binding heterogeneity: the larger are the stability constants, the more significant is the occupation of binding sites by metal ions, therefore widening the range of metal binding affinities and metal complex stability constants. This is in line with the data given in Tables 1 and 3 .

According to the results collected in Table 3, heterogeneity in metal binding increases with temperature for both types of particles investigated. For the results obtained at $15 \mathrm{mM}$ ionic strength, 
$\Delta \alpha$ values are -within experimental error- similar at $15^{\circ} \mathrm{C}$ and $25^{\circ} \mathrm{C}$ for both particle types investigated and significantly decrease at $35^{\circ} \mathrm{C}$. Increasing temperature leads to a loss of water from the collapsing (dilute) part of the particles shell as a result of attractive interactions between hydrophobic isopropyl groups of the polymer chains. Although DLS measurements suggest only a small change in particle shell thickness in the temperature range $15^{\circ} \mathrm{C}-35^{\circ} \mathrm{C}$ (Figure 1), local modifications of water structuration leading to the appearance of hydrophobic effects in the shell cannot be excluded. The resulting shell shrinking, even partial, may also promote cooperative binding effects and thus, the formation of multidentate complexes. Accordingly, with increasing temperature, affinity of metal ions to binding sites will tend to be more heterogeneous and more differentiated along the polymer chains within the particle volume. At $100 \mathrm{mM}, \Delta \alpha$ values also decrease with increasing temperature for both types of particles. We emphasize that for $\mathrm{p}(\mathrm{MA}-\mathrm{N})$ under such high salt concentration condition a significant change in the heterogeneity degree is observed between $25^{\circ} \mathrm{C}$ and $35^{\circ} \mathrm{C}$, as $\Delta \alpha$ goes from 0.70 to 0.50 . This is also accompanied by a significant $(c a .15 \mathrm{~nm}$ ) shrinking of the shell (Figure 1). This feature confirms the aforementioned explanation that invokes the impact of the modification of the hydrophobic/hydrophilic balance in the shell phase induced by an increase in temperature, along with a structural change of the shell on the heterogeneity in metal complexation. As demonstrated in our previous SANS study, ${ }^{28}$ the temperature-driven collapse of the dilute polymer phase at the periphery of the particles causes a few $\mathrm{nm}$ increase of the thickness of the condensed shell phase located in the vicinity of the particle core. Accordingly, the structural arrangement of the polymeric shell at $35^{\circ} \mathrm{C}$ may significantly differ from that prevailing at $15^{\circ} \mathrm{C}$ or $25^{\circ} \mathrm{C}$, which in turn may impact the binding properties of the functional sites located close to the particle core surface as reflected by the corresponding $\Delta \alpha$ values.

As an intermediate conclusion, results indicate that metal binding by fine tuneable stimuliresponsive core-shell nanoparticles is dictated by structural and conformational changes of the soft particulate interphase following changes in temperature and medium ionic strength. Small variations in particle size triggered by changes in temperature and/or ionic strength lead to distinct spatial confinements of metal binding sites distributed within the shell, and causes therewith significant modulations of metal complex stability and metal binding heterogeneity. In the next section, we further elaborate a methodology that makes it possible to estimate the location of metal complexes within the particle volume from proper analysis of the dependence of the measured particulate metal complex stability constant on solution ionic-strength. Results are further discussed in connection with the particle shell structure.

\section{Defining the location of the metal binding region within the particle shell}

The dependence of the particle-metal complex stability constant on ionic strength (Table 1) putatively stems from corresponding changes in the activity coefficient of metal ions in solution. This contribution can be evaluated by comparing the ratio of the measured $K_{\mathrm{ML}}$ values at $15 \mathrm{mM}$ and 100 
$\mathrm{mM}$ solution ionic strength with the ratio between activity coefficients of the metal ions at these two ionic strengths. The values of $\log \gamma_{\mathrm{M}^{2+}}$ are -0.44 at $I=100 \mathrm{mM}$ and -0.21 at $I=15 \mathrm{mM}$, with $\gamma_{\mathrm{M}^{2+}}$ the activity coefficient of metal ions as determined from Davies equation. ${ }^{47}$ This provides a ratio $\log \left(\gamma_{\mathrm{M}^{2+}}\right)^{15 \mathrm{mM}} / \log \left(\gamma_{\mathrm{M}^{2+}}\right)^{100 \mathrm{mM}}$ of 0.48 within the temperature range $15-35^{\circ} \mathrm{C}$, which is well below the value of $c a .2$ obtained for the $K_{\mathrm{ML}}$ ratio, irrespective of the temperature conditions and the type of particle considered (see Table 1). It is then clear that modification of the activity coefficients of metal ions in solution is not sufficient to explain the change in metal complex stability constants within the tested range of ionic strengths.

Pending a refined analysis of the contribution of intraparticulate electrostatics to metal-complex stability, identification of the nature of metal binding region within the shell is possible. Namely, variation of $K_{\mathrm{ML}}$ with changing solution salinity may be governed by two distinct processes: (i) the classical Eigen-Fuoss mechanism ${ }^{14,48}$ applicable for the formation of a complex between metal ion and (isolated) binding site, both interacting via coulombic forces or (ii) the metal complexation within or at the surface of a Donnan phase corresponding to the particle shell. To discriminate between these processes, let us first formulate the electrostatic contribution to the apparent stability constant of a ML complex via the relationship

$K_{\mathrm{ML}}=K_{\text {int }} \times f_{\text {elec }}$

, where $K_{\text {int }}$ is the intrinsic (chemical) stability constant and $f_{\text {elec }}$ a function that depends on the electrostatic potential metal ion experiences at the location of the binding site. Interpreting the variations of experimental $K_{\mathrm{ML}}$ values with increasing salt concentration from $15 \mathrm{mM}$ to $100 \mathrm{mM}$ in terms of the ratio $\left(K_{M L}\right)^{15 \mathrm{mM}} /\left(K_{M L}\right)^{100 \mathrm{mM}}=\left(f_{\text {elec }}\right)^{15 \mathrm{mM}} /\left(f_{\text {elec }}\right)^{100 \mathrm{mM}}$, we can evaluate the type of electrostatic contributions driving metal complex formation, either by considering the complexation of $M$ with loose and isolated binding sites or the binding of $M$ in a smeared-out Donnan-like phase. Each type of metal binding process corresponds to different $f_{\text {elec }}$ ratios that we detail below.

The first approach was originally developed by Eigen andFuoss. ${ }^{14,48}$ The theory, if applicable for the systems of interest in this work, implies that $\mathrm{Cd}$ binding by carboxylate groups results from an electrostatic contact leading to an ion-pair formation, mainly governed by the outer-sphere stability constant $K_{\mathrm{OS}}$. The formulation of the outer-sphere complex stability and its dependence on salinity via screened coulombic interactions are given by ${ }^{21}$ :

$K_{\mathrm{OS}}=\frac{4 \pi}{3} N_{a} a_{g}^{3} \exp \left(-\frac{z_{\mathrm{M}} z_{\mathrm{L}} e^{2}}{4 \pi \varepsilon_{0} \varepsilon_{r} k_{B} T a_{c c}}\left(1-\frac{\kappa a_{c c}}{1+\kappa a_{c c}}\right)\right)$.

This expression of $K_{\mathrm{OS}}$ results from the combination of the Fuoss equation with Debye-Huckel electrostatics for point charge (coulombic) interaction with account of charge screening by ions from background electrolyte ions. Here $z_{\mathrm{M}}$ and $z_{\mathrm{L}}$ are the charge number of the metal ion and the binding site, respectively, $\varepsilon_{0}$ and $\varepsilon_{r}$ are the vacuum dielectric permittivity and relative dielectric permittivity of the medium, respectively, with the dependence of $\varepsilon_{r}$ on temperature as reported in ${ }^{49}, a_{g}$ is the 
geometrical center-to-center distance between $\mathrm{M}$ and binding site, $a_{c c}$ is the charge center-to-center distance between $\mathrm{M}$ and binding site, and $\kappa$ is the reciprocal screening Debye layer thickness that depends on medium salt concentration according to classical Debye equation. The exponential term in eqn (7) corresponds to the searched $f_{\text {elec }}$ applicable for metal complex formation obeying Eigen-Fuoss mechanism.

In the second approach, we consider the formation of the metal complex within a Donnan volume defined by a given volume density of fixed (structural) charges it carries. Assessment of the Donnan potential in an ion-penetrable polymer structure is achieved following the work of Ohshima. ${ }^{50}$ According to DLS data (Figure 1), the shell thickness of the $\mathrm{p}(\mathrm{N}-\mathrm{AA})$ or $\mathrm{p}(\mathrm{MA}-\mathrm{N})$ particles ranges from 40 to $72 \mathrm{~nm}$ in the temperature and salinity conditions of interest in this work (the core radius is ca. $42 \mathrm{~nm}$, see ref [28]). Then, the thickness of the shell is always larger than the Debye thickness $\kappa^{-1}$ which equals to $2.4 \mathrm{~nm}$ and $0.96 \mathrm{~nm}$ at $15 \mathrm{mM}$ and $100 \mathrm{mM}$ ionic strength, respectively, which legitimates the application of Donnan representation for the particles adopted in this study under the tested conditions. The relationship between the dimensionless Donnan potential $y_{D}$ operational in the Donnan volume (dimensionless potentials $y$ are defined here by $y=F \Psi / R T$ with $\Psi$ the potential) and the dimensionless surface potential $y_{0}$ applying at the edge of the Donnan volume of the soft particulate ligand is given by ${ }^{50}$ :

$\left(y_{D}-y_{0}\right) \times\left\{\frac{\kappa_{m}(b-a) \cosh \left[\kappa_{m}(b-a)\right]+\left(\kappa_{m}^{2} a b-1\right) \sinh \left[\kappa_{m}(b-a)\right]}{\kappa_{m} a \cosh \left[\kappa_{m}(b-a)\right]+\sinh \left[\kappa_{m}(b-a)\right]}\right\}=2 \kappa b \sinh \left(\frac{y_{0}}{2}\right) \times\left[1+\frac{2}{\kappa b \cosh ^{2}\left(y_{0} / 4\right)}+\right.$

$\left.\frac{8 \ln \left[\cosh \left(y_{0} / 4\right)\right]}{(\kappa b)^{2} \sinh ^{2}\left(y_{0} / 2\right)}\right]^{1 / 2}$

where $a$ is the particle core radius, $b$ the radius of the whole particle including shell compartment, $\kappa_{m}$ $=\kappa \sqrt{\cosh y_{D}}$ with $y_{D}=\sinh ^{-1}\left(\rho_{0} / 2 z_{\mathrm{L}} c_{\infty} F\right)$ and $\rho_{0} / F$ the space charge density in the Donnan phase and $c_{\infty}$ the 1:1 bulk electrolyte concentration. Solving the transcendental eqn (8) yields to the evaluation of $y_{0}$. Here, the relevant $f_{\text {elec }}$ factor to be implemented in eqn (6) is given by the exponential term $\exp \left(-z_{\mathrm{M}} y_{r}\right)$ where $y_{r}$ is set to $y_{D}$ or $y_{0}$, which corresponds to metal complexation in the particle Donnan volume or at the particle outer shell surface. Accordingly, eqn (8) provides values of $\left(K_{M L}^{y_{r}}\right)^{15 \mathrm{mM}} /\left(K_{M L}^{y_{r}}\right)^{100 \mathrm{mM}}$ for different electrostatic scenarios, the relevance of which can be apprehended upon comparison with the ratio between stability constants measured at 15 and $100 \mathrm{mM}$. The analysis is performed below at 15,25 and $35^{\circ} \mathrm{C}$.

In Figure $4 \mathrm{~A}$, we report the ratio between experimental $K_{\mathrm{ML}}$ values at $15 \mathrm{mM}$ and $100 \mathrm{mM}$ as a function of temperature. Results are compared with the ratios $\left(K_{\mathrm{OS}}\right)^{15 \mathrm{mM}} /\left(K_{\mathrm{OS}}\right)^{100 \mathrm{mM}}$ and $\left(K_{\mathrm{ML}}^{y_{r}}\right)^{15 \mathrm{mM}} /\left(K_{\mathrm{ML}}^{y_{r}}\right)^{100 \mathrm{mM}}$, calculated according to eqn (7) and eqns (6) and (8), respectively. The parameters used for the determination of $K_{\mathrm{OS}}$ or $K_{\mathrm{ML}}^{y_{r}}$ are detailed in the figure caption. As shown in Figure 4 , the impact of electrostatics on $K_{\mathrm{OS}}$ is very limited as the ratio $\left(K_{\mathrm{OS}}\right)^{15 \mathrm{mM}} /\left(K_{\mathrm{OS}}\right)^{100 \mathrm{mM}}$ 
remains close to unity under the various conditions relevant in this work. In contrast, within the Donnan electrostatic framework, higher values of $\left(K_{\mathrm{ML}}^{y_{r}}\right)^{15 \mathrm{mM}} /\left(K_{M L}^{y_{r}}\right)^{100 \mathrm{mM}}$ are found, which underlies a larger effect of electrostatics on $K_{\mathrm{ML}}^{y_{r}}$.

In Figure 4A, we first analyse the situation where the space charge densities $\rho_{0}$ required for the determination of $K_{\mathrm{ML}}^{y_{r}}$ are directly evaluated from protolytic titration data detailed in [28] and corrected here for shell volume variation measured by DLS at $\mathrm{pH} 7.5$ (Figure 1). The obtained values of $\rho_{0} / F$ are approximatively $-300 \mathrm{mM}$ and $-270 \mathrm{mM}$ for $\mathrm{p}(\mathrm{N}-\mathrm{AA})$ and $\mathrm{p}(\mathrm{MA}-\mathrm{N})$, respectively. Using these $\rho_{0} / F$ values, Figure 4A reveals two kinds of metal binding scenarios depending on the particle type considered. While metal binding by $\mathrm{p}(\mathrm{MA}-\mathrm{N})$ particles is consistent with the Donnan approach, metal binding by $\mathrm{p}(\mathrm{N}-\mathrm{AA})$ particles satisfactorily agrees with Eigen-Fuoss mechanism. From this result, it turns out that for $\mathrm{p}(\mathrm{N}-\mathrm{AA})$ particle type where reactive sites are significantly exposed to the solution side of the particle/solution interphase due their location at the outer particle shell periphery, ML formation likely follows the binding of metal ions to charges carried by loose polymer chains located within the dilute shell component identified in [28]. For p(MA-N) particles, the magnitude of the variation in $K_{\mathrm{ML}}$ ratio with changing solution salinity invokes a metal partitioning at the shell interphase phase dominated by a Donnan effect. Such a difference between the two particles emphasizes once again an impact of the charge location within the shell on metal complexation. It is emphasized that the above comparison between experimental results and theory does not involved any adjustable parameter.

Closer inspection of Figure 4A reveals that experimental results obtained with $\mathrm{p}(\mathrm{MA}-\mathrm{N})$ as particulate ligand are better reproduced if considering the surface potential $y_{0}$ instead of the bulk Donnan potential for the evaluation of the electrostatic contribution $f_{\text {elec }}$ of the complex stability constant. This indicates that metal ions in the shell are preferably positioned at the outer particle shell rather than in the particle volume domain, which is a priori not consistent with the location of the charges expectedly present in the vicinity of the core. We add however that the density of charges determined from potentiometric titration is taken constant throughout the whole shell phase and therefore the representation of the electric potential profile throughout the shell may be inappropriate as we then neglect the preferential confinement of the charges close to the core surface. Since the radial distribution of charges density across the shell was suggested in [28] as being sharp and highly dense near the core, we attempted to confront our metal binding results obtained with p(MA-N) following the same Donnan approach but with considering a reduced shell thickness that is comparable in size to that of the condensed shell phase, c.a. $10 \mathrm{~nm}$ thick. ${ }^{28}$ However, the so-recalculated $K_{\mathrm{ML}}^{y_{r}}$ ratios are then shifted towards higher values due to concomitant enhancement of the volume charge density, and consequently the deviation from experimental data is more pronounced (not shown). To achieve an acceptable fit of data, decreasing value of $\rho_{0}$ is necessarily required. The guess of both the volume charge density and the shell thickness parameters without precise knowledge of the charge distribution 
obviously becomes speculative. Despite these uncertainties, results given in Figure 4A suggest that only a fraction of the reactive sites, located at the edge of the Donnan volume, significantly contributes to metal complexation.
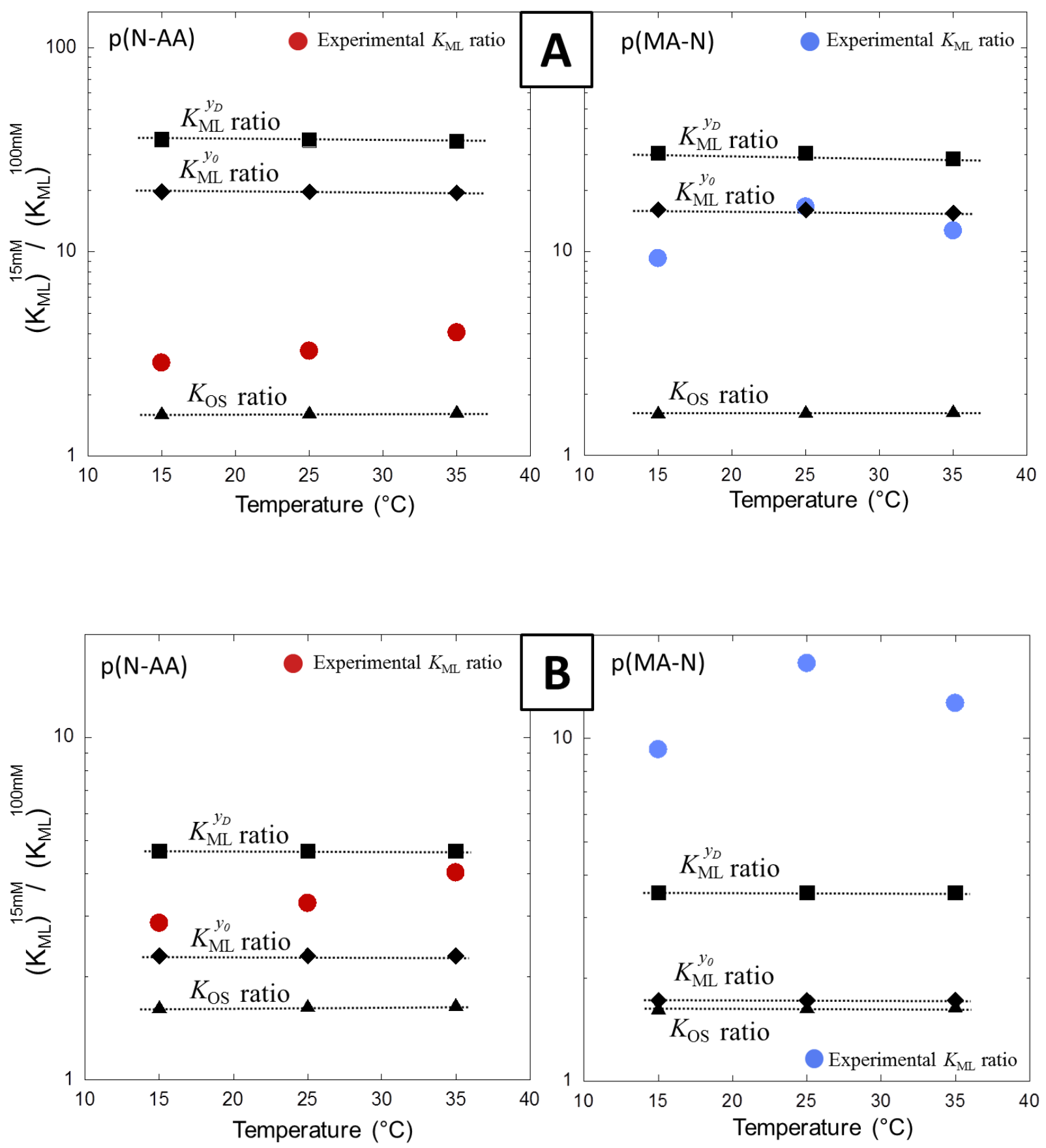

Figure 4: Ratio between experimental stability constants obtained at $15 \mathrm{mM}$ and $100 \mathrm{mM}$ as a function of temperature (indicated). The data are compared to ratios of $K_{\mathrm{OS}}$ or $K_{\mathrm{ML}}^{y_{r}}$ (with $y_{r}=y_{D}$ or $y_{0}$ ) values at $15 \mathrm{mM}$ and $100 \mathrm{mM}$ as evaluated according to eqn (7) and eqns (6),(8), respectively (dotted lines). For the calculation of $K_{\mathrm{OS}}$ , the parameters $a_{c c}$ and $a_{g}$ were set to $5 \AA .{ }^{6}$ For the determination of $K_{\mathrm{ML}}^{y_{r}}$, the required space charge density was determined from protolytic titration (panel A) and electrokinetic analyses (panel B) detailed in [28] (see text for more details). 
In Figure 4B, we treat an alternative scenario where volume charge densities are derived from previously published electrokinetic data. ${ }^{28}$ This provides ten-fold lower values of $\rho_{0}$ compared to those stemming from potentiometric titrations due to the reduced peripheral shell region probed by the electroosmotic flow under electrophoresis conditions. ${ }^{28}$ The electrophoretic particle mobility is indeed determined by the only fraction of carboxylic groups located within the peripheral electrokineticallyactive zone of the shell whose thickness is few times the Debye length. ${ }^{13,51}$ Following this approach, at pH 7.5 the maximum charge densities achievable are $-32 \mathrm{mM}$ and $-25 \mathrm{mM}$ for $\mathrm{p}(\mathrm{N}-\mathrm{AA})$ and $\mathrm{p}(\mathrm{MA}-\mathrm{N})$ particles, respectively. The corresponding implications on ML stability constant ratio are given in Figure 4B. Obviously, the tested scenario is not consistent with metal binding by p(MA-N) particles as experimental values now become significantly higher than the resulting $K_{M L}^{y_{r}}$ ratios. Electrokinetic data then provide a severe underestimation of the volume density of carboxylic groups involved in metal complex formation because they do not involve the contribution of the charges located in the vicinity of the particle core that are not detectable under electrokinetic conditions. On the opposite, a good agreement is found for the $\mathrm{p}(\mathrm{N}-\mathrm{AA})$ case for which experimental values are now intermediate between scenarios where ML formation occurs at the particle surface and in the Donnan 'volume'. Recalling that the charge density considered here pertains to the carboxylic groups located at the outer shell periphery, we can conclude that metal ions experience electrostatic field in the outer domain of the shell, which is again consistent with the expected location of the metal binding carboxylic moieties. Even more interesting is the variation of the $K_{\mathrm{ML}}$ ratios with temperature, which suggests a transition between particle surface complexation to a 'volume' complexation upon increasing temperature from $15^{\circ} \mathrm{C}$ to $35^{\circ} \mathrm{C}$. This finding is in line with particle shell shrinking that leads to a reduction in the number of chains freely protruding from the particle surface toward the outer electrolyte solution, and thus to a better definition of a step-like electrostatic Donnan transition from bulk particle to outer electrolyte solution. As a last result, we evaluated the intrinsic component of the $K_{\mathrm{ML}}$ constants using values collected in Table 1 and the electrostatic contribution identified according to the above analysis. We obtained $\log K_{\text {int }}=0.40,0.48$ and 0.75 for $\mathrm{p}(\mathrm{N}-\mathrm{AA})$ and $\log K_{\mathrm{int}}=0.50,0.51$ and 0.90 for $\mathrm{p}(\mathrm{MA}-\mathrm{N})$ at $15,25,35^{\circ} \mathrm{C}$, respectively. For a given temperature, the $K_{\text {int }}$ values are relatively close between the two particles, evidencing a similar intrinsic chemical contribution to the $\mathrm{Cd}$ binding with $\mathrm{p}(\mathrm{N}-\mathrm{AA})$ and p(MA-N) particulate ligands. In agreement with the discussion provided in section 4.3 , the increase of $K_{\text {int }}$ with temperature reflects the enhancement of binding cooperative effects triggered by structural changes.

Overall interpretation of metal binding properties of responsive core-shell nanoparticles 


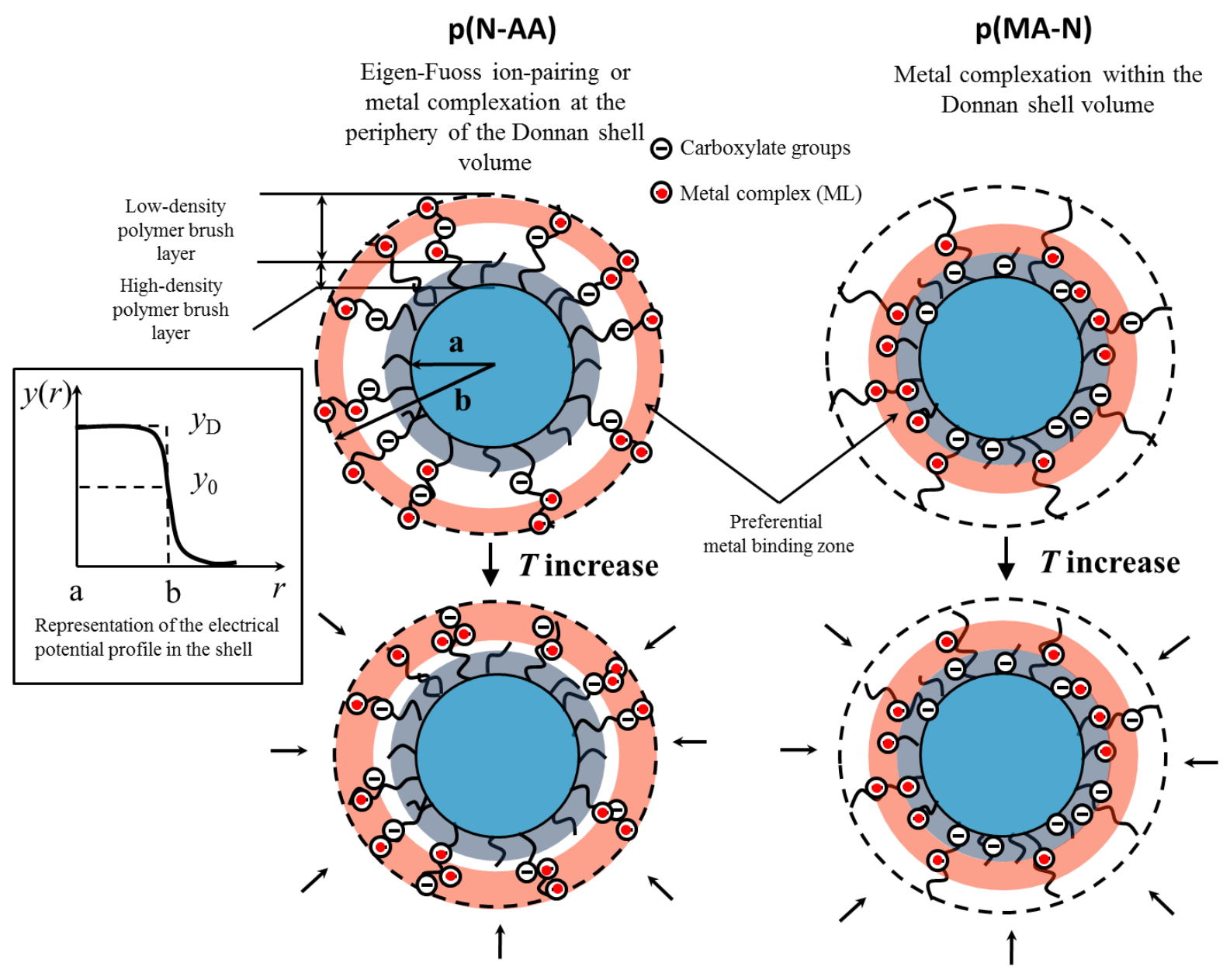

Figure 5: Schemes representing the preferential locations of metal binding in the shell compartments of $p(N-$ AA) and $\mathrm{p}(\mathrm{MA}-\mathrm{N})$ particulate ligands according to temperature (see text for more details).

To summarize the results, we report in Figure 5 schemes representing the location of metal complexes within the shell of $\mathrm{p}(\mathrm{N}-\mathrm{AA})$ and $\mathrm{p}(\mathrm{MA}-\mathrm{N})$ particles as a function of temperature. At $15^{\circ} \mathrm{C}$, Cd-binding by $\mathrm{p}(\mathrm{N}-\mathrm{AA})$ particles occurs at the level of the reactive sites preferentially located at the outer region of the shell, leading to a relatively weak heterogeneity in metal binding $(\Delta \alpha=0.66$ and 0.80 at $15 \mathrm{mM}$ and $100 \mathrm{mM}$ solution ionic strength, respectively). This scenario is supported either by an ion-pairing complex formation or by metal binding at the edge of a polyelectrolyte Donnan volume. At $35^{\circ} \mathrm{C}$, the formation of metal complexes takes place in a more condensed Donnan volume. Under such temperature condition, the heterogeneity in metal binding is more pronounced as compared to the situation at $15^{\circ} \mathrm{C}$. This is attributed to the shrinking of the polymeric shell that brings metal binding sites closer to each other, which enhances the cooperativity in metal binding process and therewith the heterogeneity in metal complex forms and stability. In the case of $\mathrm{p}(\mathrm{MA}-\mathrm{N})$ particles, a scenario of ion-pair complexation mediated by coulombic interactions clearly fails to describe experimental data. The electrostatic contribution to metal binding by carboxylate moieties is better defined by a Donnan approach despite the difficulty to unambiguously define the applicable charge distribution. It should be noted that $\mathrm{p}(\mathrm{MA}-\mathrm{N})$ particles exhibit higher stability complexes, which evidences efficient 
cooperativity in metal complexation leading to formation of multidentate complexes as compared to the situation met with $\mathrm{p}(\mathrm{N}-\mathrm{AA})$ particulate ligands. Cd complexation by $\mathrm{p}(\mathrm{MA}-\mathrm{N})$ particles thus occurs preferentially within the particle shell, which leads to broader distribution in metal complex stability constants compared to that obtained with $\mathrm{p}(\mathrm{N}-\mathrm{AA})$ particles.

\section{Conclusions.}

The results reported in this work on the thermodynamics of Cd binding by core-shell p(MA-N) and $\mathrm{p}(\mathrm{N}-\mathrm{AA})$ nanoparticles give a rich overview about the effects of (i) reactive particulate shell structure and (ii) charges location within the shell on metal complexation processes. Despite weak $\mathrm{Cd}$ interactions with carboxylate groups, we demonstrate here that features (i) and (ii) above dramatically affect metal complex stability and heterogeneity in metal binding. The latter mainly stems from the dehydration of the shell through the increase in temperature and the associated spatial condensation and shrinking of the polymeric shell. The results obtained here with a well-controlled and characterized colloidal particle raise several questions regarding the relevance of current thermodynamic framework commonly adopted for quantifying metal binding by environmental colloids, which includes ubiquitous humic matter adsorbed on e.g. mineral particles, or various types of bacterial cell walls, all consisting of soft polymeric material. Indeed, these models (e.g. NICADonnan, ${ }^{52} \mathrm{WHAM}^{53}$ or mineral surfaces (e.g. Dzombak-Morel model, ${ }^{54} \mathrm{CD}-\mathrm{Music}^{55}$ ) mostly ignore details of soft structure organization, stimuli (temperature, $\mathrm{pH}$ or salinity)-mediated structure modifications and how these affect particle-metal complex stability and intraparticulate metal binding heterogeneity. This work provides a first basis for understanding these effects and it further details a methodology for evaluating the relevant intraparticulate metal binding region from application of refined soft particle electrostatic models.

\section{Outline of the ESI content :}

Section A. Additional details on AGNES technique.

Section B. Supporting Figure S1 displaying supplementary SSCP results for Cd binding to p(N-AA) particles at an ionic strength of $100 \mathrm{mM}$ and $\mathrm{Cd}$ (II) binding to $\mathrm{p}(\mathrm{MA}-\mathrm{N})$ particles at $15 \mathrm{mM}$ and 100 $\mathrm{mM}$ solution ionic strength.

Acknowledgements: This work was partially supported by Fundação para a Ciência e a Tecnologia (FCT-Portugal) and COMPETE (FEDER), project PTDC/CTM-POL/3698/2014. 


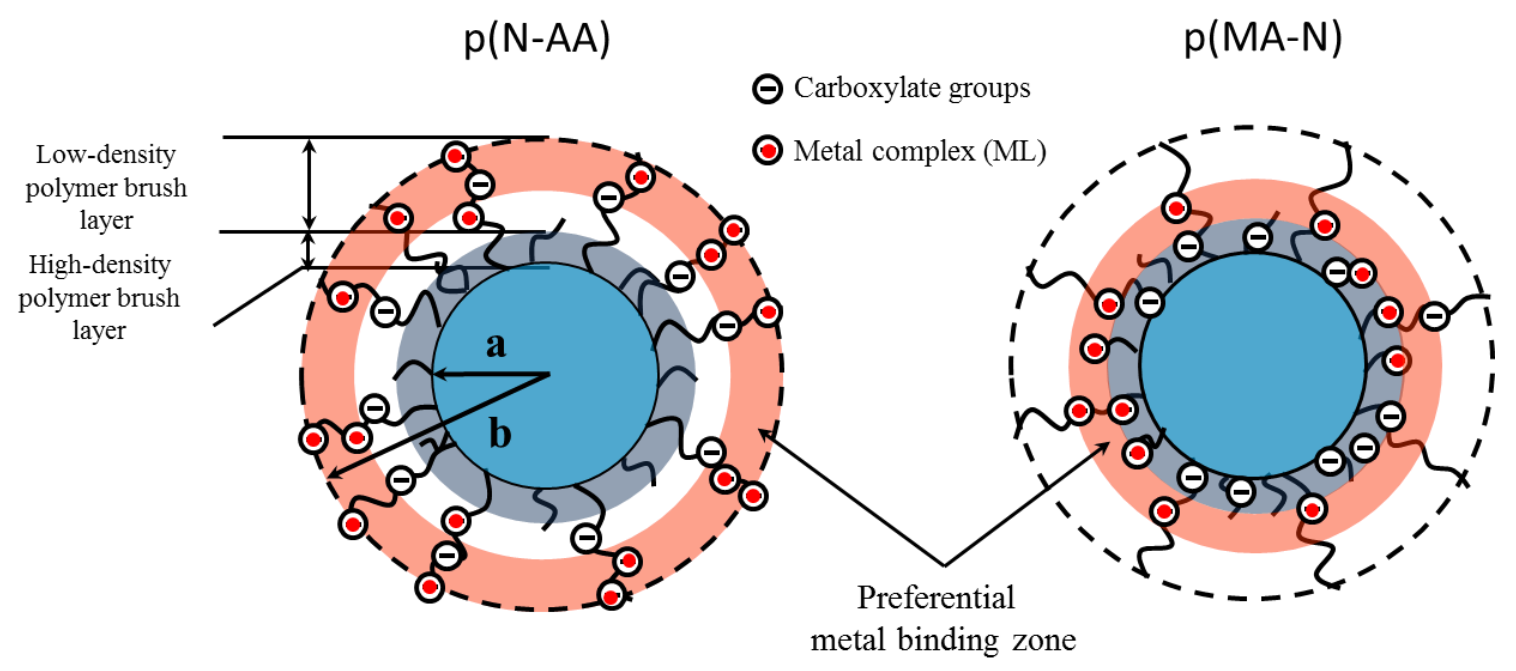




\section{References}

1 J. Buffle, R. A. Chalmers, M. R. Masson and D. Midgley, Complexation reactions in aquatic systems: an analytical approach, E. Horwood, 1988.

2 M. Filella, in Environmental colloids and particles: behaviour, separation and characterisation, eds. K. J. Wilkinson and J. R. Lead, John Wiley \& Sons, Chichester, West Sussex, UK, 2007, pp. 17-93.

3 G. E. Batley, in Trace Element Speciation in Surface Waters and Its Ecological Implications, ed. G. G. Leppard, Springer US, 1983, pp. 17-36.

4 L.-S. Wen, P. Santschi, G. Gill and C. Paternostro, Mar. Chem., 1999, 63, 185-212.

5 H. P. van Leeuwen and J. Buffle, Environ. Sci. Technol., 2009, 43, 7175-7183.

6 J. P. Pinheiro, M. Minor and H. P. van Leeuwen, Langmuir, 2005, 21, 8635-8642.

7 H. P. van Leeuwen, J. Buffle, J. F. L. Duval and R. M. Town, Langmuir, 2013, 29, 10297-10302.

8 J. Duval, K. Wilkinson, H. Van Leeuwen and J. Buffle, Environ. Sci. Technol., 2005, 39, 64356445.

9 E. Dague, J. Duval, F. Jorand, F. Thomas and F. Gaboriaud, Biophys. J., 2006, 90, 2612-2621.

10 J. Langlet, F. Gaboriaud, C. Gantzer and J. F. L. Duval, Biophys. J., 2008, 94, 3293-3312.

11 F. Gaboriaud, M. L. Gee, R. Strugnell and J. F. L. Duval, Langmuir, 2008, 24, 10988-10995.

12 E. Rotureau, F. Thomas and J. F. L. Duval, Langmuir, 2007, 23, 8460-8473.

13 J. F. L. Duval and F. Gaboriaud, Curr. Opin. Colloid Interface Sci., 2010, 15, 184-195.

14 M. Eigen, Pure Appl. Chem., 1963, 6, 97-116.

15 R. M. Town, J. Buffle, J. F. L. Duval and H. P. van Leeuwen, J. Phys. Chem. A, 2013, 117, $7643-$ 7654.

16 H. P. van Leeuwen, R. M. Town and J. Buffle, Langmuir, 2011, 27, 4514-4519.

17 R. M. Town, J. F. L. Duval, J. Buffle and H. P. van Leeuwen, J. Phys. Chem. A, 2012, 116, 64896496.

18 H. P. van Leeuwen, J. Buffle and R. M. Town, Langmuir, 2012, 28, 227-234.

19Z. Zhang, J. Buffle and D. Alemani, Environ. Sci. Technol., 2007, 41, 7621-7631.

20 J. F. L. Duval, J. P. Pinheiro and H. P. van Leeuwen, J Phys Chem A, 2008, 112, 7137-7151.

21 J. F. L. Duval, J. Phys. Chem. A, 2009, 113, 2275-2293.

22 J. F. L. Duval and S. Qian, J. Phys. Chem. A, 2009, 113, 12791-12804.

23 J. Lluís Garcés, F. Mas, J. Cecília, E. Companys, J. Galceran, J. Salvador and J. Puy, Phys. Chem. Chem. Phys., 2002, 4, 3764-3773.

24 C. Rey-Castro, S. Mongin, C. Huidobro, C. David, J. Salvador, J. L. Garcés, J. Galceran, F. Mas and J. Puy, Environ. Sci. Technol., 2009, 43, 7184-7191.

25 E. Rotureau and H. P. van Leeuwen, J Phys Chem A, 2008, 112, 7177-7184.

26 J. A. Leenheer, G. K. Brown, P. MacCarthy and S. E. Cabaniss, Environ. Sci. Technol., 1998, 32, 2410-2416.

27 N. Fatin-Rouge, A. Milon, J. Buffle, R. R. Goulet and A. Tessier, J. Phys. Chem. B, 2003, 107, $12126-12137$.

28 J. R. S. Martin, I. Bihannic, C. Santos, J. P. S. Farinha, B. Demé, F. A. M. Leermakers, J. P. Pinheiro, E. Rotureau and J. F. L. Duval, Langmuir, 2015, 31, 4779-4790.

29 J. P. Pinheiro, L. Moura, R. Fokkink and J. P. S. Farinha, Langmuir, 2012, 28, 5802-5809.

30 J. Galceran, E. Companys, J. Puy, J. Cecilia and J. L. Garces, J. Electroanal. Chem., 2004, 566, 95109.

31 R. M. Town, Environ. Sci. Technol., 2008, 42, 4014-4021.

32 R. M. Town and H. P. van Leeuwen, Aust. J. Chem., 2004, 57, 983-992.

33 H. P. van Leeuwen, R. M. Town, J. Buffle, R. F. M. J. Cleven, W. Davison, J. Puy, W. H. van Riemsdijk and L. Sigg, Environ. Sci. Technol., 2005, 39, 8545-8556.

34 J. Pinheiro and H. van Leeuwen, J. Electroanal. Chem., 2004, 570, 69-75.

35 D. D. DeFord and D. N. Hume, J. Am. Chem. Soc., 1951, 73, 5321-5322.

36 V. G. Levich, Physicochemical hydrodynamics., Prentice-Hall, Englewood Cliffs, N.J., 1962.

37 H. van Leeuwen and R. Town, J. Electroanal. Chem., 2002, 536, 129-140.

38 R. M. Town and H. P. van Leeuwen, Electroanalysis, 2004, 16, 458-471.

39 R. M. Town and H. P. van Leeuwen, J. Electroanal. Chem., 2003, 541, 51-65. 
40 H. Van Leeuwen and R. Town, Environ. Sci. Technol., 2003, 37, 3945-3952.

41 S. P. C. Alves, J. P. Pinheiro, J. P. S. Farinha and F. A. M. Leermakers, J. Phys. Chem. B, 2014, 118, 3192-3206.

42 J.-P. S. Sagou, E. Rotureau, F. Thomas and J. F. L. Duval, Colloids Surf. Physicochem. Eng. Asp., 2013, 435, 16-21.

43 Monterroso S.C.C., Carapuca H.M., Simao J.E.J. and Duarte A.C., Anal. Chim. Acta, 2004, 503, 203-212.

44 G. Anderegg, Pure Appl. Chem., 1982, 54.

45 C. G. Sinn, R. Dimova and M. Antonietti, Macromolecules, 2004, 37, 3444-3450.

46 H. D. Burrows, D. Costa, M. L. Ramos, M. da G. Miguel, M. H. Teixeira, A. A. C. C. Pais, A. J. M. Valente, M. Bastos and G. Bai, Phys. Chem. Chem. Phys., 2012, 14, 7950.

47 F. M. M. Morel and J. G. Hering, Principles and Applications of Aquatic Chemistry, WileyInterscience, New York, 1st edition., 1993.

48 R. M. Fuoss, J. Am. Chem. Soc., 1958, 80, 5059-5061.

49 C. Malmberg and A. Maryott, J. Res. Natl. Bur. Stand., 1956, 56, 1-8.

50 H. Ohshima, J. Colloid Interface Sci., 2008, 323, 92-97.

51 M. Moussa, C. Caillet, R. M. Town and J. F. L. Duval, Langmuir, 2015, 31, 5656-5666.

52 E. Tipping, Comput. Geosci., 1994, 20, 973-1023.

53 D. G. Kinniburgh, C. J. Milne, M. F. Benedetti, J. P. Pinheiro, J. Filius, L. K. Koopal and W. H. Van Riemsdijk, Environ. Sci. Technol., 1996, 30, 1687-1698.

54 D. A. Dzombak and F. M. M. Morel, Surface Complexation Modeling: Hydrous Ferric Oxide, John Wiley \& Sons, 1990.

55 T. Hiemstra and W. H. Van Riemsdijk, J. Colloid Interface Sci., 1996, 179, 488-508. 